DEPT. OF MATH. UNIV. OF OSLO

PURE MATHEMATICS NO 24

ISSN $0806-2439 \quad$ DECEMBER 2008

\title{
MIXED FINITE ELEMENT METHODS FOR LINEAR VISCOELASTICITY USING WEAK SYMMETRY
}

\author{
MARIE E. ROGNES AND RAGNAR WINTHER
}

\begin{abstract}
Small deformations of a viscoelastic body are considered through the linear Maxwell and Kelvin-Voigt models in the quasi-static equilibrium. A robust mixed finite element method, enforcing the symmetry of the stress tensor weakly, is proposed for these equations on simplicial tessellations in two and three dimensions. A priori error estimates are derived and numerical experiments presented. The approach can be applied to general models for linear viscoelasticity and thus offers a unified framework.
\end{abstract}

\section{INTRODUCTION}

Viscoelastic materials are characterized by their ability to display both viscous and elastic behaviour. Most real-life solids demonstrate some viscoelastic properties, and these effects may be particularly important when considering synthetic polymers or biological materials such as muscles or soft tissue. In this paper, we revisit the fundamental models for small deformation viscoelasticity in the quasistatic equilibrium, with the purpose of deriving a robust and flexible mixed finite element method. In particular, we consider the analysis of, and numerics for, two basic models and provide arguments for how the setup extends to generalized viscoelastic models.

The established theory for linear elasticity provides a sound starting point for the study of linear viscoelasticity. In this work, we shall rely on known results on the stability and robustness of mixed finite element methods for linear elasticity. The classical approach to linear isotropic elasticity consists of solving Navier's equations for the displacement $u$ over a domain $\Omega$ :

$$
\operatorname{div}(2 \mu \varepsilon(u)+\lambda \operatorname{div} u I)=g \quad \text { in } \Omega,
$$

where $g$ is some prescribed body force. The Lamé coefficients $\mu$ and $\lambda$ relate to the stiffness and compressibility of the material respectively. These equations can be solved numerically by for instance a standard finite element method giving optimal order error estimates for $u$ using continuous piecewise vector polynomials.

However, the quantity of primary physical interest is often the stress and pure displacement methods will yield stress approximations of lower order accuracy. Furthermore, it is well-known that standard discretizations based on the formulation of (1.1) is not robust with regard to the material parameters. The effect is that the method performs poorly in the incompressible and nearly incompressible case, i.e. as $\lambda \rightarrow \infty$. Alternative approaches are therefore eligible. A mixed formulation involving the stress tensor in addition to the displacement address the afore issues. For this approach, the main obstacle has been the construction of stable pairs of finite element spaces. Non-composite families of such elements have been established only in the recent years, cf. $[1,4,8]$ for simplicial tesselations in two and three dimensions. However, the complexity of these may seem prohibitive: The lowest order element space on a simplex has 21 degrees of freedom in two dimensions and 156 in three dimensions. 
Partially as a remedy when suitable finite element spaces were not known, and partially in order to avoid the cost of such elements, a further extension has been considered. Instead of enforcing the symmetry of the stress through the element space directly, it can be enforced weakly by an additional equation and an associated Lagrange multiplier. This idea dates back to the 1970 's, originally suggested by Fraijs de Veubeke [24], and various stable element spaces have been presented in later works, including $[3,5,6,20,21,39]$. This approach enables the use of simpler finite element spaces for the stresses. Hence, since the multiplier associated with the symmetry constraint can be approximated in a relatively small element space, the total complexity can be reduced. We shall pursue this approach in this exposition.

For linear viscoelasticity, where the stress-strain relationship may be non-local, stress-displacement methods are especially advantageous. For instance, for the linear viscoelastic Maxwell model, the quasi-static equations can take the form:

$$
\begin{aligned}
A_{1} \dot{\sigma}(t)+A_{0} \sigma(t) & =\varepsilon(\dot{u}(t)) \\
\operatorname{div} \sigma(t) & =g(t),
\end{aligned}
$$

where the superimposed dot gives the time-derivative and $A_{0}, A_{1}$ are fourth-order material tensors. These equations are suited for a mixed stress-displacement method. In contrast, an elimination of the stress relies on an inversion of the stress-strain relation. The resulting formulation would involve an integro-differential equation for the displacements. The equivalence of the differential and the integral models was discussed by Gurtin and Sternberg in [25].

Most of the numerical work on linear viscoelasticity in the late 1980's and 1990's focused on such hereditary integral formulations, reflecting the inherent interpretation of viscoelastic materials as materials with memory. These terms refer to the property that the stress does not depend on the strain, or its rate of change, pointwise in time, but rather on the history of the strain evolution. For the linear theory, this idea, along with a Boltzmann superposition principle, gives integro-differential models in the form of Volterra integrals. The stress can then be expressed as an integral operator of the strain, typically of the form

$$
\sigma(t)=C(t) \varepsilon(u(0))+\int_{0}^{t} C(t-s) \varepsilon(\dot{u}(s)) \mathrm{d} s
$$

where $C$ is a time-dependent fourth order material tensor. For the afore Maxwell model in one dimension, $C$ takes the form $C(t)=A_{1}^{-1} e^{-A_{1} t / A_{0}}$. The stress can then again be eliminated to yield a pure displacement integral formulation. Numerical methods for such problems thus involve approximations in time and space of $u$.

We shall not pursue these formulations further in this paper, and therefore simply remark that it has been extensively studied, including from a numerical point of view. A detailed mathematical review for the integral formulations can be found in the monograph [19]. Shaw et al. presented a series of papers, [35, 36, 37, 38], including a priori and a posteriori error estimates, using continuous and discontinuous Galerkin finite elements for the spatial and time discretization respectively. Discontinuous Galerkin methods for the spatial discretization have been studied by Rivière et al. [33]. The integral kernel can be extended to fractional order time derivatives and treated by similar techniques [2].

The differential form of the constitutive equations has regained some of its popularity over the last decade. These formulations typically require the introduction of internal variables corresponding to internal state variables, such as elastic or viscous contributions to the stress or strain. The generalized Maxwell models have been a common starting point for most of the studies. In [34], Rivière et al. follow Johnson and Tessler [29] with regard to introducing internal stresses in $L^{2}\left(\Omega ; \mathbb{R}^{d \times d}\right)$ while seeking the displacement in $H^{1}\left(\Omega ; \mathbb{R}^{d}\right), d=2,3$. Idesman et al. [28] consider 
a converse approach introducing internal variables for the strains, in contrast to the stresses.

To our knowledge, there is only a handful of papers concerned with the analysis of mixed finite element methods for this type of formulations. Le Tallec and Ravachol considered a mixed finite element method inspired by the Stokes equations for the Maxwell model and its non-linear extensions including extensions for viscoelastic flow [30]. The more recent paper [10], thoroughly treats the dynamic (generalized) Zener model by approximating the symmetric internal stresses in addition to the displacements. However, the element spaces of the latter require regular cubical partitions and thus lay restrictions on the computational domain. No study for general simplicial partitions in two and three dimensions, flexibly treating both basic and generalized models in their differential form, seems to have been undertaken.

1.1. Main results. The main aim of this paper is to propose a robust and accurate mixed finite element method for generalized linear viscoelasticity models on general domains in two and three dimensions.

To this end, we revisit the basic Kelvin-Voigt and Maxwell models in their differential form. We consider a mixed finite element method for the spatial discretization inspired by the family of elements introduced by Arnold et al. [6]. For each polynomial degree $k$, these consist of piecewise discontinuous polynomials of order $k-1$ for the displacements and the auxiliary Lagrange multiplier, and the $\mathrm{BDM}_{k}[14,31]$ elements, that is, polynomials of order $k$ with inter-element normal continuity, for the stresses. We prove stability of the continuous solutions and their spatially discrete counterparts with regard to data along with deriving error estimates of the order $\mathcal{O}\left(h^{k}\right)$ for sufficiently smooth solutions. Furthermore, we indicate how the discretization and analysis can be extended to generalized linear viscoelasticity models of the form:

$$
\begin{aligned}
A_{V}^{j} \sigma_{j}+A_{E}^{j} \dot{\sigma}_{j} & =\varepsilon(\dot{u}) \quad j=0, \ldots, n-1, \\
\operatorname{div} \sum_{j=0}^{n-1} \sigma_{j} & =g .
\end{aligned}
$$

In all, this intends to show how mixed finite elements for linear elasticity are available and suitable also for linear viscoelasticity.

1.2. Outline. The organization of this paper is as follows: We provide a brief derivation of, along with equations for, the viscoelastic models of interest in Section 2. In Section 3, we carefully derive weak formulations of the models, explain the relation to the linear elasticity equations and provide energy estimates demonstrating stability in time. Section 4 deals with the spatial discretization and a priori error estimates for this discretization, while the fully discrete system is considered in Section 5. For the time-discretization, we mainly refer to previous results on implicit differentiation schemes for differential-algebraic equations. Finally, we provide numerical examples in Section 6 before we conclude in Section 7.

\section{Viscoelastic MODELS}

In this section, we shall describe a class of models representing viscoelastic behaviour. The material presented here is classical in many senses. However, this review is targeted at providing motivation for the choice of viewpoint in Section 3 . In particular, we shall focus on the derivation of stress-strain relations and point out less classical, alternative formulations. The reader can find a more thorough discussion of viscoelastic behaviour and modelling among the references [17, 19, 23, 32, 40]. 
The classical continuum modelling of solids is based on balance of linear momentum in combination with an appropriate constitutive equation. For the quasi-static state, the former equation takes the form

$$
\operatorname{div} \sigma=g,
$$

where $\sigma$ is the stress tensor and $g$ a body force. The material characteristics of the solid must be reflected in a constitutive model relating the stress tensor to the strain (and possibly rates of strain) of the body. In the small-deformation framework, the linearized strain tensor $\varepsilon$ is defined in terms of the displacement $u$ by the relation: $2 \varepsilon(u)=\operatorname{grad} u+\operatorname{grad} u^{T}$. We shall focus on constitutive equations for viscoelastic materials in the subsequent paragraphs.

Various viscoelastic behaviour patterns may be illustrated in one dimension by combinations of springs and dashpots, representing elastic and viscous factors respectively. The corresponding constitutive laws may be derived from the compatibility and equilibrium conditions of the physical systems represented. Generalizations to two or three dimensions can be performed by considering deviatoric and volumetric contributions separately.

The simplest manner in which to schematically construct a viscoelastic model is to combine one elastic and one viscous component either in series or in parallel. The resulting models are known as the Maxwell and the Kelvin-Voigt model or element respectively. Note that these are clearly simplicial and only capable of describing viscoelastic behaviour partially. However, these basic models provide buildingblocks for the construction of more realistic models. For instance, an arbitrary number of Maxwell elements in parallel, or analogously, a serial combination of Kelvin-Voigt elements could model the complex behaviour displayed by viscoelastic materials within the linear regime. With this in mind, we turn to the precise differential formulations of the two basic models.

Let $\sigma$ denote the total stress, $u$ the total displacement and $\varepsilon(u)$ the linearized strain as before. The subscripts $E$ and $V$ will denote elastic and viscous components respectively in this section. We begin by considering the Maxwell model. In this case, the following equations relate the elastic and viscous components of the displacement to the stress:

$$
\sigma=C_{E} \varepsilon\left(u_{E}\right), \quad \sigma=C_{V} \varepsilon\left(\dot{u}_{V}\right), \quad u=u_{E}+u_{V},
$$

where $C_{E}$ and $C_{V}$ are fourth-order material tensors. Assuming isotropy, these take the reduced form:

$$
C \tau=2 \mu \tau+\lambda \operatorname{tr} \tau I
$$

where $\mu$ and $\lambda$ are the scalar, possibly spatially varying, Lamé coefficients. We will not restrict our attention to the isotropic case in the following, though we shall assume that the tensors $C$ are invertible and independent of time. The notation $A=C^{-1}$ is used throughout.

The classical formulation of the Maxwell model, given in (2.3) below, may be obtained by inverting the stress-strain relations, differentiating the equation for the elastic contributions with respect to time and adding the resulting two constitutive equations to replace $\dot{u}_{E}+\dot{u}_{V}$ by $\dot{u}$.

$$
A_{E} \dot{\sigma}+A_{V} \sigma=\varepsilon(\dot{u})
$$

The equation (2.3) should be equipped with the initial condition

$$
\sigma(0)=\zeta \text { where } \operatorname{div} \zeta=g(0)
$$

and $g$ is as in (2.1). Note that the purely elastic case can be viewed as a special case of the Maxwell model, with $A_{V}=0$. 
For the Kelvin-Voigt model, the starting point is similar, but the compatibility conditions take a different form. We consider the same constitutive equations for the separate elastic and viscous contributions, but now the combined system reads:

$$
\sigma_{E}=C_{E} \varepsilon(u), \quad \sigma_{V}=C_{V} \varepsilon(\dot{u}), \quad \sigma=\sigma_{E}+\sigma_{V} .
$$

The classical formulation for the Kelvin-Voigt model can be formed by summing the contributions to give the equation

$$
\sigma=C_{E} \varepsilon(u)+C_{V} \varepsilon(\dot{u}) .
$$

Eliminating the stress, using (2.1), results in a pure displacement formulation:

$$
\operatorname{div}\left(C_{E} \varepsilon(u)+C_{V} \varepsilon(\dot{u})\right)=g .
$$

An alternative would be to imitate to procedure used for Maxwell: Keep the separate stress components, invert the stress-strain relations and differentiate the equation for the elastic components with respect to time. This strategy yields the equations:

$$
A_{E} \dot{\sigma}_{E}=\varepsilon(\dot{u}), \quad A_{V} \sigma_{V}=\varepsilon(\dot{u}), \quad \sigma=\sigma_{E}+\sigma_{V} .
$$

The natural initial condition is now

$$
\sigma_{E}(0)=\zeta_{E},
$$

where $\zeta_{E}$ could be calculated from an initial displacement $u(0)$ using (2.5).

The latter approach has the immediate disadvantage that additional tensorvalued variables are introduced to the system of equations. However, there are also several advantages. First, we would expect discretizations of the displacement formulation (2.6) to be wrought with the same, or more severe, problems in the nearly incompressible case as discretizations of (1.1). In contrast, the strain-stress form of the Kelvin-Voigt equations (2.7) will enable the use of robust mixed finite element methods for the stress and the displacement. Second, the mixed approach enables a unified formulation of the two models, and thus lays the foundation for flexibly treating a wide range of viscoelastic models. For these reasons, we shall pursue the formulation in (2.7). We shall conclude this section by some comments on the treatment of the generalized models.

Equations for generalized viscoelastic models may be formulated in a variety of ways; One classical formulation [16, p. 52] is the constitutive relationship

$$
\sum_{l=0}^{k} A_{l} \frac{\mathrm{d}^{l} \sigma}{\mathrm{dt}^{l}}=\sum_{l=0}^{k} B_{l} \frac{\mathrm{d}^{l} \varepsilon}{\mathrm{dt}^{l}}
$$

where $A_{l}$ and $B_{l}$ are again fourth-order material tensors. However, any viscoelastic model derived from a linear spring-dashpot combination, can be reduced to an equivalent series-parallel model [40, p. 135]. Further, the standard series-parallel models, can be reduced to a number of Maxwell elements, springs and dashpots in parallel. Such combinations, with $n$ elements in parallel, can be expressed in the form:

$$
\begin{aligned}
A_{E}^{j} \dot{\sigma}_{j}+A_{V}^{j} \sigma_{j} & =\varepsilon(\dot{u}) \quad j=0, \ldots, n-1, \\
\sigma & =\sum_{j=0}^{n-1} \sigma_{j},
\end{aligned}
$$

where $A_{E}^{j}, A_{V}^{j}$ may be zero for some $j$, along with the initial conditions $\sigma_{k}(0)=\zeta_{k}$ for $k$ such that $A_{E}^{k} \neq 0$. If $A_{E}^{j} \neq 0$ for all $j=0, \ldots, n-1$, the compatibility condition $\operatorname{div} \sum_{j} \zeta_{j}=g(0)$ applies. Note that these initial conditions are consistent with the 
initial conditions introduced above for the Maxwell and Kelvin-Voigt models. For instance, the standard linear solid model ${ }^{1}$ takes the form:

$$
\begin{aligned}
A_{E}^{0} \dot{\sigma}_{0}+A_{V}^{0} \sigma_{0} & =\varepsilon(\dot{u}), \quad A_{E}^{1} \dot{\sigma}_{1}=\varepsilon(\dot{u}), \\
\sigma & =\sigma_{0}+\sigma_{1} .
\end{aligned}
$$

The discretization approach studied in this paper can, in principle, be applied for any model in the class defined by (2.9). However, the analysis will be focused on the basic models (2.3) and (2.7). Furthermore, in Section 6, we present some numerical experiments relating to the model (2.10).

\section{WEAK FORMULATIONS AND STABILITY ESTIMATES}

The main focus of this section is to derive weak formulations for the Maxwell and the Kelvin-Voigt models. We preface this derivation by introducing appropriate notation and provide an analogy to the Hellinger-Reissner formulation for linear elasticity. We conclude the section by giving stability estimates, thus demonstrating that the weak solutions are stable with regard to the initial conditions and data.

We start by introducing some notation. Let $\Omega$ be an open, bounded domain in $\mathbb{R}^{d}, d=2,3$, with Lipschitz boundary $\partial \Omega$. We denote the space of square integrable functions on $\Omega$ by $L^{2}(\Omega)$ with inner product $\langle\cdot, \cdot\rangle$ and norm $\|\cdot\|_{0, \Omega}$. The standard Sobolev spaces $H^{m}(\Omega)$ have norm $\|\cdot\|_{H^{m}}$ and semi-norm $|\cdot|_{H^{m}}$ for $m=1,2, \ldots$. The reference to the domain $\Omega$ will be omitted when context makes it superfluous. Further, a norm without subscripts will default to the $L^{2}$ norm. If $\langle A \cdot, \cdot\rangle$ is a coercive bilinear form on $L^{2}(\Omega)$, we shall denote the induced norm by $\|\cdot\|_{A}$. The linear spaces of vectors, matrices, symmetric matrices and skewsymmetric matrices are denoted by $\mathbb{V}, \mathbb{M}, \mathbb{S}$ and $\mathbb{K}$ respectively. The space of fields on $\Omega$ with square integrable components and values in $X$, is denoted $L^{2}(\Omega ; X)$. Moreover, the subspace of $L^{2}(\Omega ; \mathbb{V})$ of vector fields with square integrable divergence is denoted by $H(\operatorname{div}, \Omega ; \mathbb{V})$ with the associated norm $\|\cdot\|_{\text {div }}$. Accordingly, the space of matrix fields with rows in $H(\operatorname{div}, \Omega ; \mathbb{V})$ is denoted $H(\operatorname{div}, \Omega ; \mathbb{M})$ and the analogous subspace of symmetric matrix fields is denoted $H(\operatorname{div}, \Omega ; \mathbb{S})$.

For the formulation of the Kelvin-Voigt model and the generalized models, we will require a product space of matrix fields, denoted by $H^{+}$, having the property that the sum is in $H(\operatorname{div}, \Omega ; \mathbb{M})$. More precisely,

$$
H^{+}=\left\{\left(\tau_{0}, \tau_{1}\right) \in L^{2}(\Omega ; \mathbb{M})^{2} \mid \tau_{0}+\tau_{1} \in H(\operatorname{div}, \Omega ; \mathbb{M})\right\} .
$$

We shall also require spaces involving time [18, p.285 ff.]. Let $X$ be a Hilbert space with norm $\|\cdot\|_{X} \cdot L^{2}(0, T ; X)$ will denote the space of strongly measurable functions $u:[0, T] \rightarrow X$ with norm

$$
\|u\|_{L^{2}(0, T ; X)}=\left(\int_{0}^{T}\|u(t)\|_{X}^{2}\right)^{1 / 2}<\infty .
$$

Differentiation with respect to time is denoted by a superposed dot. Finally, $H^{1}(0, T ; X)$ denotes the space of fields $u \in L^{2}(0, T ; X)$ such that $\dot{u} \in L^{2}(0, T ; X)$ exists in the weak sense.

3.1. Weak formulations for linear elasticity. The Hellinger-Reissner formulation of linear elasticity, with pure Dirichlet boundary conditions for the displacement, takes the form of finding $\sigma \in H(\operatorname{div}, \Omega ; \mathbb{S})$ and $u \in L^{2}(\Omega ; \mathbb{V})$ satisfying

$$
\begin{aligned}
\langle A \sigma, \tau\rangle+\langle\operatorname{div} \tau, u\rangle & =0 \quad \forall \tau \in H(\operatorname{div}, \Omega ; \mathbb{S}), \\
\langle\operatorname{div} \sigma, w\rangle & =\langle g, w\rangle \quad \forall w \in L^{2}(\Omega ; \mathbb{V}),
\end{aligned}
$$

\footnotetext{
${ }^{1}$ The standard linear solid model is equivalent to the Zener model, and was studied, along with its generalized version, in [10].
} 
where $g \in L^{2}(\Omega ; \mathbb{V})$ is a given body force and $A=A(x): \mathbb{S} \rightarrow \mathbb{S}$ is a uniformly positive definite operator. For isotropic, homogenous elastic materials with shear modulus $\mu$ and stiffness $\lambda$, the action of $A$ reduces to

$$
A \sigma=\frac{1}{2 \mu}\left(\sigma-\frac{\lambda}{2 \mu+d \lambda}(\operatorname{tr} \sigma) I\right) .
$$

The derivation of this formulation relies on the invertibility of the elastic stressstrain relationship and the symmetry of the stress tensor.

Following [13], the existence and uniqueness of solutions to these equations depend on the existence of a positive constant $\alpha$ such that

$$
\langle A \tau, \tau\rangle \geq \alpha\|\tau\|_{\text {div }} \quad \text { for all } \tau \in Z,
$$

where

$$
Z=\left\{\tau \in H(\operatorname{div}, \Omega ; \mathbb{S}) \mid\langle\operatorname{div} \tau, w\rangle=0 \text { for all } w \in L^{2}(\Omega ; \mathbb{V})\right\} .
$$

Since $\operatorname{div} H(\operatorname{div}, \Omega ; \mathbb{S}) \subset L^{2}(\Omega ; \mathbb{V})$, the uniform positive definiteness of the operator $A$ on $L^{2}(\Omega ; \mathbb{S})$ guarantees condition (3.4). In addition, there must, and does, exist a positive constant $\beta$ such that

$$
\|v\|_{0} \leq \beta \sup _{\tau \in H(\operatorname{div}, \Omega ; \mathbb{S})} \frac{|\langle\operatorname{div} \tau, v\rangle|}{\|\tau\|_{\operatorname{div}}} .
$$

The latter condition is usually referred to as the inf-sup condition.

The positive definiteness of the isotropic $A$, defined by (3.3), fails as $\lambda \rightarrow \infty$. However, a uniform coercivity estimate can be established under the additional requirement that $\int \operatorname{tr} \sigma=0$. Since $A$ is also uniformly continuous in $\lambda$, this formulation makes the desired robustness in the case $\lambda \rightarrow \infty$ attainable.

Unfortunately, the construction of stable pairs of finite element spaces for the discretization of these equations has proven, in the course of four decades of research, to be nontrivial. In the last decade, stable finite element spaces, associated with a single triangulation family, have been constructed in both two and three dimensions $[1,4,8]$. These families of element spaces are advantageous in the sense that the approximation error of the stress can be separated from that of the displacement. On the other hand, their complexity, including complexity of implementation, makes other approaches eligible.

In this work, we shall pursue the alternative weak symmetry approach [24]. Instead of restricting the stress tensor space to tensor fields with symmetric values, the symmetry can be enforced by the introduction of a constraint and a Lagrange multiplier $\rho$. More precisely, the weak symmetry formulation of (3.2) reads: Find $\sigma \in H(\operatorname{div}, \Omega ; \mathbb{M}), u \in L^{2}(\Omega ; \mathbb{V})$ and $\rho \in L^{2}(\Omega ; \mathbb{K})$ satisfying

$$
\begin{aligned}
\langle A \sigma, \tau\rangle+\langle\operatorname{div} \tau, u\rangle+\langle\tau, \rho\rangle & =0 \quad \forall \tau \in H(\operatorname{div}, \Omega ; \mathbb{M}), \\
\langle\operatorname{div} \sigma, w\rangle+\langle\sigma, \eta\rangle & =\langle g, w\rangle \quad \forall w \in L^{2}(\Omega ; \mathbb{V}), \eta \in L^{2}(\Omega ; \mathbb{K}) .
\end{aligned}
$$

Formally, the variable $\rho$ corresponds to the skew component of the gradient of $u$ : $2 \rho=\operatorname{grad} u-\operatorname{grad} u^{T}$. On the continuous level, the formulations (3.2) and (3.6) are equivalent. However, the weakening of the symmetry constraint opens up the possibility for simpler element spaces for the stress. In the following, we shall frequently require the trilinear form of $(3.6 \mathrm{~b})$ and therefore label it here for later reference:

$$
b(\tau, w, \eta)=\langle\operatorname{div} \tau, w\rangle+\langle\tau, \eta\rangle
$$

The inf-sup condition for this formulation, corresponding to (3.5) for the strong symmetry formulation, guarantees the existence of a $\beta>0$ such that for any 
$v \in L^{2}(\Omega ; \mathbb{V})$ and $\eta \in L^{2}(\Omega ; \mathbb{K})$,

$$
\|v\|_{0}+\|\eta\|_{0} \leq \beta \sup _{\tau \in H(\operatorname{div}, \Omega ; \mathbb{M})} \frac{|b(\tau, v, \eta)|}{\|\tau\|_{\text {div }}} .
$$

The reader is referred to for example [11] for details and proofs of the afore stability assertions relating to (3.2) and (3.6).

3.2. Weak formulations for linear viscoelasticity. We now find ourselves in the position to derive weak formulations with weak symmetry for the Maxwell and Kelvin-Voigt models. The constitutive relationships are defined by (2.3) and (2.7) respectively and the system is closed by the balance of linear momentum (2.1), the initial conditions of (2.4) and (2.8) and boundary conditions. In the subsequent analysis, we shall assume natural homogenous boundary conditions, i.e. $u(t)=0$ on $\partial \Omega$ for simplicity. We introduce the velocity $v=\dot{u}$ and the rotation of the velocity $\gamma=\dot{\rho}$ as these are the more natural variables for the formulations. Both $u(t)$ and $\rho(t)$ can clearly be post-calculated given an additional initial condition for $u$.

The strain-stress form of the Maxwell constitutive equation (2.3), in combination with (2.1), yields the following weak equations after an integration by parts of the strain term.

$$
\begin{aligned}
\left\langle A_{0} \sigma, \tau\right\rangle+\left\langle A_{1} \dot{\sigma}, \tau\right\rangle+b(\tau, v, \gamma) & =0 \quad \forall \tau \in H(\operatorname{div}, \Omega ; \mathbb{M}), \\
b(\sigma, w, \eta) & =\langle g, w\rangle \quad \forall w \in L^{2}(\Omega ; \mathbb{V}), \eta \in L^{2}(\Omega ; \mathbb{K}),
\end{aligned}
$$

where $g$ is given and $b$ is defined in (3.7). We have suppressed the dependency on time in the notation of (3.9).

We proceed to consider the Kelvin-Voigt model. Multiplying (2.7) and (2.1) by test functions, integrating by parts and enforcing the symmetry of the full stress tensor weakly, give the weak formulation of the Kelvin-Voigt model in (3.10) below. The original subscripts $V$ and $E$ have been replaced by 0 and 1 respectively.

$$
\begin{array}{r}
\left\langle A_{1} \dot{\sigma}_{1}, \tau_{1}\right\rangle+\left\langle A_{0} \sigma_{0}, \tau_{0}\right\rangle+b\left(\tau_{0}+\tau_{1}, v, \gamma\right)=0 \quad\left(\tau_{0}, \tau_{1}\right) \in H^{+}, \\
b\left(\sigma_{0}+\sigma_{1}, w, \eta\right)=\langle g, w\rangle \quad w \in L^{2}(\Omega ; \mathbb{V}), \eta \in L^{2}(\Omega ; \mathbb{K}),
\end{array}
$$

where $H^{+}$is as defined by (3.1).

We further observe that a weak formulation for the generalized models defined by (2.9) can be derived in the entirely analogous manner. We enforce the symmetry of the total stress tensor $\sigma=\sum_{j} \sigma_{j}$ weakly, i.e. $\langle\sigma, \eta\rangle=0$ for all $\eta \in L^{2}(\Omega ; \mathbb{K})$, as for the Kelvin-Voigt model. The weak equations then take the form:

$$
\begin{aligned}
\left\langle A_{E}^{j} \dot{\sigma}_{j}, \tau_{j}\right\rangle+\left\langle A_{V}^{j} \sigma_{j}, \tau_{j}\right\rangle+b\left(\tau_{j}, v, \gamma\right) & =0 \quad \forall \tau_{j}, \quad j=0, \ldots, n-1, \\
b\left(\sum_{j} \sigma_{j}, w, \eta\right) & =\langle g, w\rangle \quad \forall w, \eta .
\end{aligned}
$$

These derivations demonstrate that this type of formulation is well-suited for both the basic and the generalized models. We therefore have a unified framework in place. At this point however, we let the generalized models rest in order to avoid notational overflow.

3.3. Existence and regularity of solutions. We shall give arguments for the existence of solutions to the systems of equations (3.9) and (3.10) in order to motivate the assumptions to follow.

First, consider the Maxwell model of (3.9) with the initial condition $\sigma(0)=$ $\zeta \in H(\operatorname{div}, \Omega ; \mathbb{M})$. Assume that $g \in H^{1}\left(0, T ; L^{2}(\Omega ; \mathbb{V})\right)$ and that $\operatorname{div} \zeta=g(0)$. Then, there exist $\sigma_{e} \in H^{1}(0, T ; H(\operatorname{div}, \Omega ; \mathbb{M})), u_{e} \in H^{1}\left(0, T ; L^{2}(\Omega ; \mathbb{V})\right)$ and $\rho_{e} \in H^{1}\left(0, T ; L^{2}(\Omega ; \mathbb{K})\right)$ solving the elasticity equations (3.6) with $A=A_{0}$ for each $t \in[0, T]$. Next, let $H_{0}=\{\tau \in H(\operatorname{div}, \Omega ; \mathbb{M}) \mid \operatorname{div} \tau=0\}$. Since $A_{0}, A_{1}$ are 
bounded on $H_{0}$, there exists a $\sigma_{0} \in H^{1}\left(0, T ; H_{0}\right)$ satisfying the ordinary differential equation:

$$
\left\langle A_{1} \dot{\sigma}_{0}, \tau\right\rangle+\left\langle A_{0} \sigma_{0}, \tau\right\rangle=-\left\langle A_{1} \dot{\sigma}_{e}, \tau\right\rangle \quad \tau \in H_{0}
$$

with the initial condition $\sigma_{0}(0)=\zeta-\sigma_{e}(0) \in H_{0}$. Further, the inf-sup condition (3.8), gives the existence of $v_{0}(t) \in L^{2}(\Omega ; \mathbb{V}), \gamma_{0}(t) \in L^{2}(\Omega ; \mathbb{K})$ for a.e $t$ such that

$$
\begin{aligned}
\left\langle A_{1} \dot{\sigma}_{0}, \tau\right\rangle+\left\langle A_{0} \sigma_{0}, \tau\right\rangle+b\left(\tau, v_{0}, \gamma_{0}\right) & =-\left\langle A_{1} \dot{\sigma}_{e}, \tau\right\rangle \quad \tau \in H(\operatorname{div}, \Omega ; \mathbb{M}), \\
b\left(\sigma_{0}, w, \eta\right) & =0 \quad w \in L^{2}(\Omega ; \mathbb{V}), \eta \in L^{2}(\Omega ; \mathbb{K}) .
\end{aligned}
$$

It follows that $\sigma=\sigma_{e}+\sigma_{0}, v=u_{e}+v_{0}$ and $\gamma=\rho_{e}+\gamma_{0}$ solve the Maxwell equations (3.9) for a.e $t$ and the initial condition $\sigma(0)=\zeta$ with $\operatorname{div} \sigma(0)=g$. Further, $\sigma \in H^{1}(0, T ; H(\operatorname{div}, \Omega ; \mathbb{M})), v \in L^{2}\left(0, T ; L^{2}(\Omega ; \mathbb{V})\right)$ and $\gamma \in L^{2}\left(0, T ; L^{2}(\Omega ; \mathbb{K})\right)$. This existence argument motivates Definition 3.1.

Definition 3.1 (Weak solutions of the Maxwell equations). Assume that

$$
g \in H^{1}\left(0, T ; L^{2}(\Omega ; \mathbb{V})\right), \zeta \in H(\operatorname{div}, \Omega ; \mathbb{M}), \operatorname{div} \zeta=g(0) .
$$

The fields $\sigma \in H^{1}(0, T ; H(\operatorname{div}, \Omega ; \mathbb{M})), v \in L^{2}\left(0, T ; L^{2}(\Omega ; \mathbb{V})\right), \gamma \in L^{2}\left(0, T ; L^{2}(\Omega ; \mathbb{K})\right)$ constitute a weak solution of the Maxwell equations provided (3.9) is satisfied for a.e. $t \in(0, T]$ and the initial condition $\sigma(0)=\zeta$ holds.

Uniqueness of these solutions is a consequence of Theorem 3.1 below.

We proceed to consider the Kelvin-Voigt model with the initial condition $\sigma_{1}(0)=$ $\zeta_{1} \in L^{2}(\Omega ; \mathbb{M})$ and assume that $g \in L^{2}\left(0, T ; L^{2}(\Omega ; \mathbb{V})\right)$. Solving the stationary elasticity equation

$$
\left\langle C_{1} \varepsilon(\nu), \varepsilon(v)\right\rangle=\left\langle\zeta_{1}, \operatorname{grad} v\right\rangle \quad \forall v \in H_{0}^{1}(\Omega ; \mathbb{V}),
$$

yields $\nu \in H_{0}^{1}(\Omega ; \mathbb{V})$. Next, from a weak formulation of $(2.6)$ :

$$
\left\langle C_{1} \varepsilon(u(t))+C_{0} \varepsilon(\dot{u}(t)), \varepsilon(v)\right\rangle=\langle g(t), v\rangle, \quad \forall v \in H_{0}^{1}(\Omega ; \mathbb{V}),
$$

equipped with the initial condition $u(0)=\nu$, we can deduce the existence of a solution $u \in H^{1}\left(0, T ; H^{1}(\Omega ; \mathbb{V})\right)$. In accordance with (2.5), we define

$$
\begin{aligned}
\sigma_{0}(t) & =C_{0} \varepsilon(\dot{u}(t)) \in L^{2}\left(0, T ; L^{2}(\Omega ; \mathbb{M})\right), \\
\sigma_{1}(t) & =C_{1} \varepsilon(u(t))+\zeta_{1}-C_{1} \varepsilon(\nu) \in H^{1}\left(0, T ; L^{2}(\Omega ; \mathbb{M})\right), \\
\gamma(t) & =\frac{1}{2}\left(\operatorname{grad} \dot{u}-\operatorname{grad} \dot{u}^{T}\right) \in L^{2}\left(0, T ; L^{2}(\Omega ; \mathbb{K})\right) .
\end{aligned}
$$

It follows that $\operatorname{div}\left(\sigma_{0}+\sigma_{1}\right)=g$ and $\sigma_{1}(0)=\zeta_{1}$.

Definition 3.2 (Weak solutions of the Kelvin-Voigt equations). Assume that

$$
g \in L^{2}\left(0, T ; L^{2}(\Omega ; \mathbb{V})\right), \zeta_{1} \in L^{2}(\Omega ; \mathbb{M}) .
$$

The fields $\sigma_{0} \in L^{2}\left(0, T ; L^{2}(\Omega ; \mathbb{M})\right), \sigma_{1} \in H^{1}\left(0, T ; L^{2}(\Omega ; \mathbb{M})\right)$ satisfying $\left(\sigma_{0}(t), \sigma_{1}(t)\right) \in$ $H^{+}$for a.e. $t, v \in L^{2}\left(0, T ; L^{2}(\Omega ; \mathbb{V})\right)$ and $\gamma \in L^{2}\left(0, T ; L^{2}(\Omega ; \mathbb{K})\right)$ constitute a weak solution of the Kelvin-Voigt equations provided (3.10) is satisfied for a.e $t \in(0, T]$, and the initial condition $\sigma_{1}(0)=\zeta_{1}$ holds.

These solutions are indeed unique cf. Theorem 3.2 below.

3.4. Stability estimates. The two formulations (3.9), (3.10) can be viewed as evolutionary problems subject to a constraint. The two theorems closing this section provide stability estimates for the Maxwell and the Kelvin-Voigt equations respectively, thus demonstrating stability in time. The estimates rely on two main factors. First, the inf-sup condition (3.8) holds for the spaces $H(\operatorname{div}, \Omega ; \mathbb{M})$ and $L^{2}(\Omega ; \mathbb{V}) \times L^{2}(\Omega ; \mathbb{K})$. Second, the assumption that the operators $A_{j}: L^{2}(\Omega ; \mathbb{M}) \rightarrow$ 
$L^{2}(\Omega ; \mathbb{M})$ are uniformly positive definite and continuous, i.e. there exist positive constants $\alpha_{j}$ and $c_{j}$ such that

$$
\left\langle A_{j} \sigma, \sigma\right\rangle \geq \alpha_{j}\|\sigma\|_{0}^{2}, \quad\left\langle A_{j} \sigma, \tau\right\rangle \leq c_{j}\|\sigma\|_{0}\|\tau\|_{0} .
$$

We shall make this assumption here and throughout. As a consequence, each $A_{j}$ induces a norm equivalent to the $L^{2}$ norm: $\left\langle A_{j} \tau, \tau\right\rangle=\|\tau\|_{A_{j}}^{2}$.

For the Maxwell equations (3.9), we start by observing that if there is no applied body force, i.e. $g=0$, then

$$
\frac{1}{2} \frac{\mathrm{d}}{\mathrm{dt}}\|\sigma\|_{A_{1}}^{2}+\|\sigma\|_{A_{0}}^{2}=0 .
$$

Using the equivalence of the norms induced by $A_{0}$ and $A_{1}$ and Grönwall's inequality, we obtain the estimate:

$$
\|\sigma(t)\|_{A_{1}}^{2} \leq e^{-\frac{2 \alpha_{0}}{c_{1}} t}\|\sigma(0)\|_{A_{1}}^{2}
$$

In other words, the energy, measured in the $A_{1}$-norm of the stress, decays exponentially from its initial state. The situation with a constant body force, $\dot{g}=0$, can also be reduced to a system with $g=0$, and we can therefore derive a similar estimate in that case. The case $g \neq 0$ is covered in the following theorem.

Theorem 3.1 (Stability estimates for Maxwell). Let $(\sigma, v, \gamma)$ be a weak solution of the Maxwell equations. There exists a positive constant $c$ such that

$$
\|\sigma(t)\|_{A_{1}}^{2} \leq e^{-\frac{\alpha_{0}}{c_{1}} t}\|\zeta\|_{A_{1}}^{2}+c \int_{0}^{t} e^{-\frac{\alpha_{0}}{c_{1}}(t-s)}\left(\|g(s)\|^{2}+\|\dot{g}(s)\|^{2}\right) \mathrm{d} s
$$

and

$$
\|v(t)\|^{2}+\|\gamma(t)\|^{2}+\|\dot{\sigma}(t)\|^{2} \leq c\left(\|\dot{g}(t)\|^{2}+\|\sigma(t)\|^{2}\right)
$$

for a.e. $t \in[0, T]$.

Proof. Let $(\sigma(t), v(t), \gamma(t))$ satisfy (3.9) for a.e. $t$. In order to obtain estimates for the stress, we use $\tau=\sigma(t)$ in (3.9a) and apply (3.9b) to find that

$$
\|\sigma(t)\|_{A_{0}}^{2}+\frac{1}{2} \frac{\mathrm{d}}{\mathrm{dt}}\|\sigma(t)\|_{A_{1}}^{2}=-\langle g, v\rangle .
$$

Clearly, in order to bound the stress, we need a bound for the velocity $v$. Using Cauchy-Schwartz for the norms induced by $A_{0}$ and $A_{1}$ and the inf-sup condition of (3.8) with constant $\beta$, it follows that

$$
\|v(t)\|_{0}+\|\gamma(t)\|_{0} \leq \beta\left(c_{0}^{1 / 2}\|\sigma\|_{A_{0}}+c_{1}^{1 / 2}\|\dot{\sigma}\|_{A_{1}}\right) .
$$

Next, using $\tau=\dot{\sigma}(t)$ in (3.9a), and (3.9b) differentiated with respect to time, give an analogy to $(3.13)$ for $\dot{\sigma}$ :

$$
\left\langle A_{0} \sigma, \dot{\sigma}\right\rangle+\|\dot{\sigma}(t)\|_{A_{1}}^{2}=-\langle\dot{g}, v\rangle .
$$

Together with (3.14), this gives the desired estimate for $\dot{\sigma}$ in terms of $\sigma$.

$$
\|\dot{\sigma}(t)\|_{A_{1}}^{2} \leq c\left(\|\sigma(t)\|_{A_{0}}^{2}+\|\dot{g}(t)\|^{2}\right) .
$$

This estimate and (3.14) give (3.12). Furthermore, combining this estimate with (3.13) and (3.14), we obtain

$$
\frac{\alpha_{0}}{2 c_{1}}\|\sigma(t)\|_{A_{1}}^{2}+\frac{1}{2} \frac{\mathrm{d}}{\mathrm{dt}}\|\sigma(t)\|_{A_{1}}^{2} \leq c\left(\|g(t)\|^{2}+\|\dot{g}\|^{2}\right) .
$$

Hence, (3.11) follows by Grönwall's inequality.

Corresponding estimates can be derived for the Kelvin-Voigt equations and are summarized in the following theorem. 
Theorem 3.2 (Stability estimates for Kelvin-Voigt). Let $\left(\sigma_{0}, \sigma_{1}, v, \gamma\right)$ be a weak solution of the Kelvin-Voigt equations. There exists a positive constant $c$ such that

$$
\left\|\sigma_{1}(t)\right\|_{A_{1}}^{2}+\int_{0}^{t}\left\|\sigma_{0}(s)\right\|_{A_{0}}^{2}+\|v(s)\|^{2}+\|\gamma(s)\|^{2} \mathrm{~d} s \leq\left\|\zeta_{1}\right\|_{A_{1}}^{2}+c \int_{0}^{t}\|g(s)\|^{2} \mathrm{~d} s .
$$

for $t \in[0, T]$.

Proof. The proof is straightforward: Letting $\tau_{0}=\sigma_{0}$ and $\tau_{1}=\sigma_{1}$ in (3.10a) and using (3.10b), we obtain the relation

$$
\frac{1}{2} \frac{\mathrm{d}}{\mathrm{dt}}\left\|\sigma_{1}\right\|_{A_{1}}^{2}+\left\|\sigma_{0}\right\|_{A_{0}}^{2}=-\langle g, v\rangle .
$$

An estimate for the velocity and the rotation follows from (3.8):

$$
\|v\|_{0}+\|\gamma\|_{0} \leq \beta \sup _{\tau}\|\tau\|_{\text {div }}^{-1}|b(\tau, v, \gamma)|=\beta \sup _{\tau}|| \tau\left\|_{\text {div }}^{-1}\left|\left\langle A_{0} \sigma_{0}, \tau\right\rangle\right| \leq c|| \sigma_{0}\right\|_{A_{0}} .
$$

Combining this with (3.15) gives the final result.

We pause to remark that although we focus on the weak symmetry formulation in this paper, the strong symmetry approach also yields a meaningful base for discretization. The previous energy estimates clearly also hold for the latter approach with $\gamma$ ignored, due to the equivalence between the formulations at the continuous level. For the discrete stability and error estimates in the subsequent sections, the path of the proofs would be the same, and would to some extent be simplified with a strong, rather than the weak, symmetry constraint.

Now, having established the desired stability properties of the formulations, we move on to consider discretization strategies in space and time. We shall focus on the semi-discrete problem and conforming finite element discretizations in space in Section 4. The full discretizations in time and space will be considered in Section 5 .

\section{The SEMI-Discrete PRoblem AND STABILITy}

The scope of this section is to consider conforming finite element spatial discretizations of the two systems of equations (3.9) and (3.10). In particular, we demonstrate that the same discretization will be appropriate for both models. We shall again start by drawing inspiration from suitable finite element discretizations of the linear elasticity equations and comment on the properties of such, before embarking on the discretization of the viscoelasticity models.

Assume that $\left\{\mathcal{T}_{h}\right\}_{h}$ is a shape-regular family of admissible, simplicial tessellations of $\Omega$, where $h$ measures the mesh size. We are interested in finite element spaces $\Sigma_{h} \subset H(\operatorname{div}, \Omega ; \mathbb{M}), V_{h} \subset L^{2}(\Omega ; \mathbb{V})$ and $Q_{h} \subset L^{2}(\Omega ; \mathbb{K})$ subordinate to this tessellation. From the stationary theory of mixed finite element methods [13], we know that the discrete spaces yield a stable discretization of a given weak formulation if the Brezzi conditions hold. For the weak symmetry formulation of the linear elasticity equations (3.6), these conditions are the discrete equivalents of (3.4) and (3.8), and take the following form: For the spaces $\Sigma_{h}, V_{h}$ and $Q_{h}$, there must exist positive constants $\alpha$ and $\beta$, independent of $h$, such that

$$
\begin{aligned}
\langle A \tau, \tau\rangle & \geq \alpha\|\tau\|_{\text {div }}^{2} \quad \forall \tau \in Z_{h}, \\
\|w\|+\|\eta\| & \leq \beta \sup _{\tau \in \Sigma_{h}} \frac{|b(\tau, w, \eta)|}{\|\tau\|_{\text {div }}} \quad \forall w \in V_{h}, \eta \in Q_{h},
\end{aligned}
$$

where $b(\tau, w, \eta)=\langle\operatorname{div} \tau, w\rangle+\langle\tau, \eta\rangle$ as before and the kernel $Z_{h}$ is defined as

$$
Z_{h}=\left\{\tau \in \Sigma_{h} \mid b(\tau, w, \eta)=0 \quad \forall w \in V_{h}, \eta \in Q_{h}\right\} .
$$


There exists a multitude of finite element spaces satisfying these conditions. A selection of such can be sampled from the references $[3,5,6,24,21,39]$ and the survey [20]. In the following analysis, we shall assume that the spaces $\Sigma_{h}, V_{h}$ and $Q_{h}$ satisfy conditions (4.1) and (4.2) and additionally are such that

$$
\operatorname{div} \Sigma_{h} \subset V_{h} .
$$

Let $P_{h}$ denote the $L^{2}$ projection from $L^{2}(\Omega ; \mathbb{K})$ onto $Q_{h}$ and, with a minor abuse of notation, from $L^{2}(\Omega ; \mathbb{V})$ onto $V_{h}$. We let $\Pi_{h}$ be a projection onto $\Sigma_{h}$ such that

$$
\left\langle\operatorname{div}\left(\tau-\Pi_{h} \tau\right), w\right\rangle=0 \quad \text { for all } w \in V_{h} .
$$

We shall assume that the projection $\Pi_{h}$ is bounded, i.e. that there exists a constant $c$ such that

$$
\left\|\Pi_{h} \tau\right\|_{\text {div }} \leq c\|\tau\|_{\text {div }} \quad \text { for all } \tau \in H(\operatorname{div}, \Omega ; \mathbb{M}) .
$$

Note that the canonical projection onto $\Sigma_{h}$, defined by the degrees of freedom, is typically not bounded on $H(\operatorname{div}, \Omega ; \mathbb{M})$ and thus does not satisfy (4.5). However, bounded projections, satisfying (4.5), can be constructed through smoothing, cf. [7, $15]$.

4.1. Two stable sets of element spaces. We shall describe two families of element spaces, namely those introduced by Arnold et al. [6] and Falk [20], both satisfying the conditions (4.1), (4.2) and (4.3) above. The lowest order element spaces of these families were also suggested by Farhloul and Fortin [21]. The former family of spaces will mainly be used for the numerical experiments in Section 6.

The particular finite element spaces $\Sigma_{h}, V_{h}$ and $Q_{h}$ introduced by Arnold et al. are as follows: The lowest order elements are the combination of linear vector polynomials with continuity of normal components over inter-element facets for the stress, and piecewise constants for the velocity and the rotation approximations. The element spaces generalize to arbitrary polynomial degree; Let $\mathcal{P}_{k}\left(\mathcal{T}_{h}\right)$ denote the space of discontinuous $k$ 'th order polynomials defined on the tessellation $\mathcal{T}_{h}$ of $\Omega \subset \mathbb{R}^{d}, d=2,3$, and $\mathrm{BDM}_{k}$ denote the $k$ 'th order vector polynomials with continuous normal components over inter-element facets as introduced by Brezzi et al. [14] and extended by Nedelec [31] to three dimensions. With this notation, the $k$ 'th order elasticity elements, for $k \geq 1$, are:

$$
\Sigma_{h, k}=\operatorname{BDM}_{k}\left(\mathcal{T}_{h} ; \mathbb{V}\right), \quad V_{h, k}=\mathcal{P}_{k-1}\left(\mathcal{T}_{h} ; \mathbb{V}\right), \quad Q_{h, k}=\mathcal{P}_{k-1}\left(\mathcal{T}_{h} ; \mathbb{K}\right)
$$

For these element spaces, we have the following interpolation estimates for the projections $P_{h}^{k}$ and $\Pi_{h}^{k}$ :

$$
\begin{aligned}
\left\|\tau-\Pi_{h}^{k} \tau\right\|_{0} & \leq c h^{m+1}|\tau|_{H^{m+1}}, \\
\left\|\operatorname{div}\left(\tau-\Pi_{h}^{k} \tau\right)\right\|_{0} & \leq c h^{m}|\operatorname{div} \tau|_{H^{m}}, \\
\left\|p-P_{h}^{k} p\right\|_{0} & \leq c h^{m}|p|_{H^{m}},
\end{aligned}
$$

for $1 \leq m \leq k$.

For the weak symmetry formulation of the elasticity equations, these interpolation properties carry over to the approximation properties of the finite element discretization, with the exception that the error of the stress approximation cannot be split from that of the rotation, since skw $\Sigma_{h} \not \subset Q_{h}$. Hence, the higher-order $L^{2}$ interpolation error of the stress is not expected to be conserved.

Another stable family of element spaces, introduced by Falk [20] for the lowest order case, uses the same spaces $\Sigma_{h}$ and $V_{h}$ as of (4.6), but the space of piecewise 
constants for the rotation $Q_{h}$ is replaced by continuous piecewise linears $\tilde{Q}_{h}$. In general, we let

$$
\tilde{Q}_{h, k}=\mathcal{P}_{k}\left(\mathcal{T}_{h} ; \mathbb{K}\right) \cap C^{0}\left(\mathcal{T}_{h} ; \mathbb{K}\right)
$$

In this case, the interpolation error of the rotation is of the same order as the $L^{2}$ error of the stress interpolation. We can thus expect to retain the higher order $L^{2}$ estimates for the stress approximation.

Having discussed stable and accurate finite element spaces for discretizations of the elasticity equations, we now turn to the questions of stability and spatial a priori error estimates for the Maxwell and the Kelvin-Voigt models. Attention is paid to the Maxwell model in Section 4.2 and to the Kelvin-Voigt model in Section 4.3. The techniques involved in the following are fairly standard and we rely on the results for the stationary elasticity equations.

For ease of reading, we give the results applied to the element spaces (4.6) here. Let the subscript $h$ indicate discrete solutions and assume sufficient smoothness of the domain and the data, all which will be made precise in the subsequent sections. Then, for the Maxwell model, we will show that

$$
\left\|\sigma_{h}(t)-\Pi_{h}^{k} \sigma(t)\right\|_{0}+\left\|v_{h}(t)-P_{h}^{k} v(t)\right\|_{0}+\left\|\gamma_{h}(t)-P_{h}^{k} \gamma(t)\right\|_{0} \leq c_{T} h^{k},
$$

for a.e. $t \in[0, T]$. For the Kelvin-Voigt model,

$$
\begin{aligned}
\left\|\sigma_{1, h}(t)-\Pi_{h}^{k} \sigma_{1}(t)\right\|_{0} & +\int_{0}^{t}\left\|\sigma_{0, h}(s)-\Pi_{h}^{k} \sigma_{0}(s)\right\|_{0} \mathrm{~d} s \\
& +\int_{0}^{t}\left\|v_{h}(s)-P_{h}^{k} v(s)\right\|_{0}+\left\|\gamma_{h}(s)-P_{h}^{k} \gamma(s)\right\|_{0} \mathrm{~d} s \leq c_{T} h^{k} .
\end{aligned}
$$

4.2. Semi-discretization of the Maxwell model. For the Maxwell equations, the natural finite element spaces correspond directly to those of the elasticity equations: $\Sigma_{h} \subset H(\operatorname{div}, \Omega ; \mathbb{M}), V_{h} \subset L^{2}(\Omega ; \mathbb{V})$ and $Q_{h} \subset L^{2}(\Omega ; \mathbb{K})$. The spatially discretized equations follow immediately:

$$
\begin{array}{r}
\left\langle A_{0} \sigma_{h}(t), \tau\right\rangle+\left\langle A_{1} \dot{\sigma}_{h}(t), \tau\right\rangle+b\left(\tau, v_{h}(t), \gamma_{h}(t)\right)=0, \\
b\left(\sigma_{h}(t), w, \eta\right)=\langle g(t), w\rangle,
\end{array}
$$

for all $\tau \in \Sigma_{h}, w \in V_{h}$ and $\eta \in Q_{h}$ and for a.e $t \in(0, T]$. We also enforce the discrete initial condition $\sigma_{h}(0)=\zeta_{h} \in \Sigma_{h}$ Assuming that $\left\langle\operatorname{div} \zeta_{h}-g(0), w\right\rangle=0$ for all $w \in V_{h}$, we have semi-discrete solutions $\sigma_{h} \in H^{1}\left(0, T ; \Sigma_{h}\right), v_{h} \in L^{2}\left(0, T ; V_{h}\right)$ and $\gamma_{h} \in L^{2}\left(0, T ; Q_{h}\right)$. We observe that, if the spaces $\Sigma_{h}, V_{h}$ and $Q_{h}$ are such that the discrete Brezzi conditions (4.1) and (4.2) hold, the energy estimates for the continuous formulation carry over to the semi-discrete formulation. Thus, Theorem 3.1 also holds, with the obvious modifications, for the semi-discrete solutions.

In the subsequent error analysis however, stability estimates for the discrete equations with additional source terms will be required:

$$
\begin{array}{r}
\left\langle A_{0} \sigma_{h}(t), \tau\right\rangle+\left\langle A_{1} \dot{\sigma}_{h}(t), \tau\right\rangle+b\left(\tau, v_{h}(t), \gamma_{h}(t)\right)=\langle F(t), \tau\rangle \\
b\left(\sigma_{h}(t), w, \eta\right)=\langle G(t), \eta\rangle
\end{array}
$$

For clarity of presentation, such estimates are presented in the following lemma. Clearly, the lemma also holds for the continuous solutions with additional source terms.

Lemma 4.1 (Discrete stability with source terms for Maxwell). Assume that $G \in H^{1}\left(0, T ; L^{2}(\Omega ; \mathbb{M})\right), F \in L^{2}\left(0, T ; L^{2}(\Omega ; \mathbb{M})\right)$ and that $\left(\sigma_{h}, v_{h}, \gamma_{h}\right)$ solve (4.9) 
for a.e. $t \in(0, T]$. Then there exists a constant $c>0$ such that

$$
\begin{aligned}
& \left\|\sigma_{h}(t)\right\|_{A_{1}}^{2} \leq e^{-c t}\left\|\sigma_{h}(0)\right\|_{A_{1}}^{2}+c \int_{0}^{t} e^{-c(t-s)} K(s) \mathrm{d} s, \\
& \left\|v_{h}(t)\right\|^{2}+\left\|\gamma_{h}(t)\right\|^{2}+\left\|\dot{\sigma}_{h}(t)\right\|^{2} \leq c\left(\|F(t)\|^{2}+\|\dot{G}(t)\|^{2}+\left\|\sigma_{h}(t)\right\|^{2}\right),
\end{aligned}
$$

where $K(s)=\|F(s)\|^{2}+\|G(s)\|^{2}+\|\dot{G}(s)\|^{2}$.

Proof. Let $\tau=\sigma_{h}(t), w=v_{h}(t), \eta=\gamma_{h}(t)$ in (4.9). We obtain the equation

$$
\left\|\sigma_{h}(t)\right\|_{A_{0}}^{2}+\frac{\mathrm{d}}{\mathrm{dt}} \frac{1}{2}\left\|\sigma_{h}(t)\right\|_{A_{1}}^{2}=\left\langle F(t), \sigma_{h}(t)\right\rangle-\left\langle G(t), \gamma_{h}(t)\right\rangle
$$

The discrete inf-sup condition (4.2) gives a bound for the velocity and rotation:

$$
\left\|v_{h}\right\|+\left\|\gamma_{h}\right\| \leq c\left(\left\|\sigma_{h}\right\|+\left\|\dot{\sigma}_{h}\right\|+\|F\|\right) .
$$

Further, let $\tau=\dot{\sigma}_{h}(t), w=v_{h}(t), \eta=\gamma_{h}(t)$ in (4.9) after differentiating the second equation in time. We obtain the equation

$$
\left\langle A_{0} \sigma_{h}(t), \dot{\sigma}_{h}(t)\right\rangle+\left\|\dot{\sigma}_{h}(t)\right\|_{A_{1}}^{2}=\left\langle F(t), \dot{\sigma}_{h}(t)\right\rangle-\left\langle\dot{G}(t), \gamma_{h}(t)\right\rangle .
$$

The same techniques as employed in the proof of Theorem 3.1 give the final estimates.

We are now in the position to easily derive error estimates for the semi-discrete equations. To this aim, we introduce the following standard notation: The discretization error is split into a projection error $\mathcal{E}$ and an approximation error $E$. For the stress this takes the form

$$
\sigma-\sigma_{h}=\mathcal{E}^{\sigma}-E^{\sigma}=\left(\sigma-\Pi_{h} \sigma\right)-\left(\sigma_{h}-\Pi_{h} \sigma\right) .
$$

and we have the analogies for the velocity and the rotation, in terms of the projection $P_{h}$, with superscripts $v$ and $\gamma$ respectively. Observe that

$$
b\left(\mathcal{E}^{\sigma}, w, \eta\right)=\left\langle\mathcal{E}^{\sigma}, \eta\right\rangle
$$

for all $w \in V_{h}, \eta \in Q_{h}$ by the definition of the projection $\Pi_{h}$ onto $\Sigma_{h}(4.4)$. Moreover, due to (4.3), we have that

$$
b\left(\tau, \mathcal{E}^{v}, \mathcal{E}^{\gamma}\right)=\left\langle\tau, \mathcal{E}^{\gamma}\right\rangle
$$

for all $\tau \in \Sigma_{h}$.

Let $K(t)=\left\|\mathcal{E}^{\sigma}(t)\right\|^{2}+\left\|\mathcal{E}^{\dot{\sigma}}(t)\right\|^{2}+\left\|\mathcal{E}^{\gamma}(t)\right\|^{2}$. By definition, the function $K$ is bounded by the approximation properties of the projections $\Pi_{h}$ and $P_{h}$ and of the smoothness of the exact solution $(\sigma, v, \gamma)$. As a result of the theorem below, we obtain that the full error, which is bounded by $\|E\|+\|\mathcal{E}\|$, is of the same order as long as the initial approximation is sufficiently accurate.

Theorem 4.2 (A priori error estimates for Maxwell). The approximation errors for the stress, velocity and rotation satisfy the following bounds for a.e $t \in[0, T]$ in terms of the projection errors

$$
\begin{aligned}
& \left\|E^{\sigma}(t)\right\|^{2} \leq e^{-c t}\left\|\sigma_{h}(0)-\Pi_{h} \sigma(0)\right\|_{A_{1}}^{2}+c \int_{0}^{t} e^{-c(t-s)} K(s) \mathrm{d} s \\
& \left\|E^{\dot{\sigma}}(t)\right\|^{2}+\left\|E^{v}(t)\right\|^{2}+\left\|E^{\gamma}(t)\right\|^{2} \leq c\left(\left\|E^{\sigma}(t)\right\|^{2}+K(t)\right)
\end{aligned}
$$

Proof. We omit the reference to the time $t$ for brevity in the following. We begin by subtracting the discrete Maxwell equations from the continuous ones to obtain the error equations:

$$
\begin{array}{r}
\left\langle A_{0}\left(\sigma-\sigma_{h}\right), \tau\right\rangle+\left\langle A_{1}\left(\dot{\sigma}-\dot{\sigma}_{h}\right), \tau\right\rangle+b\left(\tau, v-v_{h}, \gamma-\gamma_{h}\right)=0 \quad \forall \tau \in \Sigma_{h}, \\
b\left(\sigma-\sigma_{h}, w, \eta\right)=0 \quad \forall w \in V_{h}, \eta \in Q_{h} .
\end{array}
$$


Inserting the error decompositions (4.10), we have:

$$
\begin{gathered}
\left\langle A_{0} E^{\sigma}, \tau\right\rangle+\left\langle A_{1} E^{\dot{\sigma}}, \tau\right\rangle+b\left(\tau, E^{v}, E^{\gamma}\right)=\left\langle A_{0} \mathcal{E}^{\sigma}, \tau\right\rangle+\left\langle A_{1} \mathcal{E}^{\dot{\sigma}}, \tau\right\rangle+\left\langle\tau, \mathcal{E}^{\gamma}\right\rangle \\
b\left(E^{\sigma}, w, \eta\right)=b\left(\mathcal{E}^{\sigma}, w, \eta\right)=\left\langle\mathcal{E}^{\sigma}, \eta\right\rangle .
\end{gathered}
$$

where the last equality follows from the definition of the projection onto $\Sigma_{h}(4.11)$. The term $\left\langle\tau, \mathcal{E}^{\gamma}\right\rangle$ is a result of (4.12). It is now easy to observe that $E^{\sigma}, E^{v}$ and $E^{\gamma}$ solve the semi-discrete equations (4.9) with the right-hand side(s):

$$
F=A_{0} \mathcal{E}^{\sigma}+A_{1} \mathcal{E}^{\dot{\sigma}}+\mathcal{E}^{\gamma}, \quad G=\mathcal{E}^{\sigma}
$$

and the initial condition $E^{\sigma}(0)=\sigma_{h}(0)-\Pi_{h} \sigma(0)$. The stability estimate with additional source terms, Lemma 4.1, thus gives the error estimates.

4.3. Semi-discretization of the Kelvin-Voigt model. This subsection aims at introducing a spatial discretization of the Kelvin-Voigt model corresponding to the one introduced for the Maxwell model in the previous. Moreover, error estimates for the semi-discrete solutions are established through an analogous extended energy estimate.

Recall that for the Kelvin-Voigt equations, we seek two components of the stress: $\sigma_{0}$ and $\sigma_{1}$ such that $\sigma_{0}+\sigma_{1}=\sigma \in H(\operatorname{div}, \Omega ; \mathbb{M})$, or with the notation introduced in $(3.1),\left(\sigma_{0}, \sigma_{1}\right) \in H^{+}$. For discretization purposes, we are therefore interested in a finite dimensional product space $\Sigma_{h}^{2}$ approximating $H^{+}$. A natural choice would be to let

$$
\Sigma_{h}^{2}=\Sigma_{h} \times \Sigma_{h}
$$

The resulting spatially discretized equations are presented below in (4.14).

$$
\begin{array}{r}
\left\langle A_{1} \dot{\sigma}_{1, h}(t), \tau_{1}\right\rangle+\left\langle A_{0} \sigma_{0, h}(t), \tau_{0}\right\rangle+b\left(\tau_{0}+\tau_{1}, v_{h}(t), \gamma_{h}(t)\right)=0, \\
b\left(\sigma_{0, h}(t)+\sigma_{1, h}(t), w, \eta\right)=\langle g(t), w\rangle,
\end{array}
$$

for all $\left(\tau_{0}, \tau_{1}\right) \in \Sigma_{h}^{2}, w \in V_{h}$ and $\eta \in Q_{h}$ and for a.e $t \in(0, T]$. As usual, we enforce the discretized initial condition: $\sigma_{1, h}(0)=\zeta_{1, h} \in \Sigma_{h}$. Again, we comment that, provided that the spaces $\Sigma_{h}, V_{h}$ and $Q_{h}$ are such that (4.1) and (4.2) hold, the energy estimates for the continuous formulations carry over to the semi-discrete formulations. Thus, Theorem 3.2 also holds, with the obvious modifications, for the semi-discrete solutions. We also note that the projections constructed in [15] are bounded in $L^{2}(\Omega ; \mathbb{M}) \mapsto L^{2}(\Omega ; \mathbb{M})$ and hence the projections of the separate stress components can be defined even if each component is not in $H(\operatorname{div}, \Omega ; \mathbb{M})$.

As in the case of the Maxwell model, a stability estimate for the equations with additional source terms is a key step in deriving error estimates. Such a result is considered separately in the following lemma. We shall however, restrict our attention to source terms $F_{0}, F_{1}$ yielding the following alternative to (4.14a):

$$
\begin{aligned}
\left\langle A_{1} \dot{\sigma}_{1, h}, \tau_{1}\right\rangle+\left\langle A_{0} \sigma_{0, h}, \tau_{0}\right\rangle+b\left(\tau_{0}+\tau_{1}, v_{h}, \gamma_{h}\right) & =\left\langle\dot{F}_{1}, \tau_{1}\right\rangle+\left\langle F_{0}, \tau_{0}\right\rangle, \\
b\left(\sigma_{0, h}+\sigma_{1, h}, w, \eta\right) & =\langle G, \eta\rangle .
\end{aligned}
$$

Recall that in the derivation of the Kelvin-Voigt model, the elastic stress-strain relation corresponding to $\sigma_{1}$ was differentiated in time. The $\dot{F}_{1}$ term of $(4.15 \mathrm{a})$ takes this aspect into account.

Lemma 4.3 (Stability with source terms for Kelvin-Voigt). Let

$$
F_{0} \in L^{2}\left(0, T ; L^{2}(\Omega ; \mathbb{M})\right), F_{1} \in H^{1}\left(0, T ; L^{2}(\Omega ; \mathbb{M})\right), G \in L^{2}\left(0, T ; L^{2}(\Omega ; \mathbb{M})\right)
$$


and assume that $\left(\sigma_{0, h}, \sigma_{1, h}, v_{h}, \gamma_{h}\right)$ solve (4.15) for a.e. $t \in(0, T]$. Then there exists a positive constant $c$ such that

$$
\begin{aligned}
\left\|\sigma_{1, h}(t)\right\|^{2}+\int_{0}^{t}\left\|\sigma_{0, h}(s)\right\|^{2}+\left\|v_{h}(s)\right\|^{2}+ & \left\|\gamma_{h}(s)\right\|^{2} \mathrm{~d} s \\
& \leq c\left(I+\left\|F_{1}(t)\right\|^{2}+\int_{0}^{t} K(s) \mathrm{d} s\right),
\end{aligned}
$$

for $t \in[0, T]$, where $I=\left\|\sigma_{1, h}(0)\right\|^{2}+\left\|F_{1}(0)\right\|^{2}$ and $K(s)=\|G(s)\|^{2}+\left\|F_{0}(s)\right\|^{2}+$ $\left\|F_{1}(s)\right\|^{2}+\left\|\dot{F}_{1}(s)\right\|^{2}$.

Proof. We omit the subscripts $h$ for notational brevity in the following. First, observe that letting $\tau_{1}=-\tau_{0}=\tau$ in (4.15a) gives the identity

$$
\left\langle A_{1} \dot{\sigma}_{1}, \tau\right\rangle-\left\langle A_{0} \sigma_{0}, \tau\right\rangle=\left\langle\dot{F}_{1}-F_{0}, \tau\right\rangle
$$

for any $\tau \in \Sigma_{h}$. In particular, letting $\tau=\dot{\sigma}_{1}$, we find that

$$
\left\|\dot{\sigma}_{1}\right\|_{A_{1}}^{2} \leq c\left(\left\|\sigma_{0}\right\|_{A_{0}}^{2}+\left\|\dot{F}_{1}\right\|^{2}+\left\|F_{0}\right\|^{2}\right) \text {. }
$$

Second, let $\tau_{0}=\sigma_{0}, \tau_{1}=\sigma_{1}, w=v$ and $\eta=\gamma$ in (4.15). We are left with:

$$
\frac{1}{2} \frac{\mathrm{d}}{\mathrm{dt}}\left\|\sigma_{1}\right\|_{A_{1}}+\left\|\sigma_{0}\right\|_{A_{0}}=-\langle G, \gamma\rangle+\left\langle\dot{F}_{1}, \sigma_{1}\right\rangle+\left\langle F_{0}, \sigma_{0}\right\rangle .
$$

Since, $\|v\|+\|\gamma\| \leq c\left(\left\|\sigma_{0}\right\|_{A_{0}}+\left\|F_{0}\right\|\right)$ by (4.2), it follows that

$$
\begin{aligned}
& \left\|\sigma_{1}(t)\right\|_{A_{1}}^{2}+\int_{0}^{t}\left\|\sigma_{0}(s)\right\|_{A_{0}}^{2} \mathrm{~d} s \leq\left\|\sigma_{1}(0)\right\|_{A_{1}}^{2} \\
& \quad+c \int_{0}^{t}\|G(s)\|^{2}+\left\|F_{0}(s)\right\|^{2} \mathrm{~d} s+\int_{0}^{t}\left\langle\dot{F}_{1}(s), \sigma_{1}(s)\right\rangle \mathrm{d} s
\end{aligned}
$$

We integrate the last term by parts to see that

$$
\int_{0}^{t}\left\langle\dot{F}_{1}(s), \sigma_{1}(s)\right\rangle \mathrm{d} s \leq\left.\left\langle F_{1}(s), \sigma_{1}(s)\right\rangle\right|_{s=0} ^{t}+\frac{1}{4 \epsilon} \int_{0}^{t}\left\|F_{1}(s)\right\|^{2} \mathrm{~d} s+\epsilon \int_{0}^{t}\left\|\dot{\sigma}_{1}(s)\right\|^{2} \mathrm{~d} s
$$

for any $\epsilon>0$. Thus, combining (4.17) with (4.16), gives the stated result.

As before, cf. (4.10), we introduce the approximation errors: $E_{h}^{\tau}=\left\|\Pi_{h} \tau-\tau_{h}\right\|$ and the projection errors: $\mathcal{E}^{\tau}=\left\|\tau-\Pi_{h} \tau\right\|$ for $\tau \in\left\{\sigma_{0}, \sigma_{1}\right\}$ and analogously for $\tau \in\{v, \gamma\}$ with $\Pi_{h}$ replaced by $P_{h}$. As a consequence of Lemma 4.3 , we obtain the following error estimates.

Theorem 4.4 (A priori error estimates for Kelvin-Voigt). Let $\left(\sigma_{0}, \sigma_{1}, v, \gamma\right)$ solve the continuous Kelvin-Voigt equations (3.10) and $\left(\sigma_{0, h}, \sigma_{1, h}, v_{h}, \gamma_{h}\right)$ be approximations satisfying the discrete equations (4.14). Then there exists $c>0$ such that

$$
\begin{aligned}
& \left\|E^{\sigma_{1}}(t)\right\|^{2}+\int_{0}^{t}\left\|E^{\sigma_{0}}(s)\right\|^{2}+\left\|E^{v}(s)\right\|^{2}+\left\|E^{\gamma}(s)\right\|^{2} \mathrm{~d} s \\
& \quad \leq c\left\|\sigma_{1, h}(0)-\Pi_{h} \sigma_{1}(0)\right\|^{2}+c\left(I(0)+I(t)+\int_{0}^{t} L(s) \mathrm{d} s\right)
\end{aligned}
$$

where $I(t)=\left\|\mathcal{E}^{\sigma_{1}}(t)\right\|^{2}+\left\|\mathcal{E}^{\rho}(t)\right\|^{2}$, and

$$
L=\left\|\mathcal{E}^{\sigma_{0}}\right\|^{2}+\left\|\mathcal{E}^{\sigma_{1}}\right\|^{2}+\left\|\mathcal{E}^{\dot{\sigma}_{1}}\right\|^{2}+\left\|\mathcal{E}^{\rho}\right\|^{2}+\left\|\mathcal{E}^{\gamma}\right\|^{2} .
$$

Again we observe that this result allows us to bound the full error in terms of the projections $\Pi_{h}$ and $P_{h}$ and the properties of the exact solution.

In conclusion, this section has treated a unified mixed finite element method for the spatial discretization of the Maxwell and the Kelvin-Voigt models. We have given a priori error estimates for the spatial discretization under the assumptions 
of saddle point stability and (4.3). We have focused on these spatial error estimates in order to present the techniques and results involved with greater clarity.

\section{FulL-DiscRETIZATION}

The aim of this section is to consider full-discretizations of the Maxwell and Kelvin-Voigt equations. The starting points are the semi-discrete formulations (4.8) and (4.14). After spatial discretization, the systems can be viewed as linear constant coefficient differential-algebraic equations (DAEs) in time. The theory of linear constant coefficient DAEs is well-developed and the monographs $[12,26]$ give a thorough summary. As this is the case, we shall not carry out an explicit analysis for different temporal discretization schemes, but rather rely on the known results for DAEs. However, we point out that it is not obvious that these time discretizations will be uniformly stable with respect to the spatial discretizations.

With regard to choice of time-discretization, there are some factors that deserve special attention. First, since the material parameters for the viscous and the elastic contributions may vary greatly, we face possibly stiff systems. Second, in thread with the previous emphasis on robustness, we aim to avoid stability conditions for the discretization parameters. These aspects make implicit time-stepping schemes attractive. Also note that since we can use an arbitrary, up to computation time, high order scheme in space, higher order schemes in time are relevant.

We shall briefly summarize the relevant concepts of and results for linear constant coefficient DAEs needed in the following, but refer to [12] for details. Linear constant coefficient DAEs take the form

$$
\mathbf{D}_{1} \dot{\mathbf{y}}+\mathrm{D}_{\mathbf{0}} \mathbf{y}=\mathbf{f}
$$

where $\mathbf{D}_{\mathbf{i}} \in \mathbb{M}^{n \times n}$ for $i=0,1$. Such systems are solvable if and only if $\lambda \mathbf{D}_{\mathbf{1}}+\mathbf{D}_{\mathbf{0}}$ is a regular pencil, i.e. if $\operatorname{det}\left(\lambda \mathbf{D}_{\mathbf{1}}+\mathbf{D}_{\mathbf{0}}\right)$ is not identically zero as a function of $\lambda$. Further, assume that the system in (5.1) is of index $\nu$ [12, p. 17]. A $k$-step implicit backward difference scheme of local order $k$ applied to (5.1) gives convergence of order $k$ after a possible initial boundary layer of thickness $(\nu-1) k+1$ time steps [12, Theorem 3.1.1]. For implicit one-step Runge-Kutta methods, matters are a bit more elaborate, and we shall again refer the reader to [12, Section 4]. In the following, we shall see how the stability conditions for the spatial discretization of the Maxwell and Kelvin-Voigt systems yields regularity and thus solvability in the DAE sense.

Consider the spatial discretizations (4.8) and (4.14) of the Maxwell and the Kelvin-Voigt equations respectively. Inserting bases for the element spaces $\Sigma_{h}, V_{h}$ and $Q_{h}$, letting $y_{h}=\left(\sigma_{j, h}, v_{h}, \gamma_{h}\right)$ and denoting the corresponding vector of finite element expansion coefficients by $\mathbf{y}$, yield following form of the equations:

$$
\mathbf{D}_{1} \dot{\mathbf{y}}+\mathrm{D}_{0} \mathbf{y}=\mathbf{f} .
$$

In particular, let $\left\{\psi_{i}\right\}_{i},\left\{\phi_{k}\right\}_{k}$ and $\left\{p_{m}\right\}_{m}$ be bases for $\Sigma_{h}, V_{h}$ and $Q_{h}$ respectively and define the element matrices

$$
\mathbf{A}_{\iota i j}=\left\langle A_{\iota} \psi_{j}, \psi_{i}\right\rangle, \quad \mathbf{B}_{k j}=\left\langle\operatorname{div} \psi_{j}, \phi_{k}\right\rangle, \quad \mathbf{C}_{m j}=\left\langle\psi_{j}, p_{m}\right\rangle .
$$

The spatial discretization of the Maxwell model takes the form

$$
\mathbf{D}_{1}=\left(\begin{array}{ccc}
\mathbf{A}_{1} & \mathbf{0} & \mathbf{0} \\
\mathbf{0} & \mathbf{0} & \mathbf{0} \\
\mathbf{0} & \mathbf{0} & \mathbf{0}
\end{array}\right) \quad \mathbf{D}_{0}=\left(\begin{array}{ccc}
\mathbf{A}_{\mathbf{0}} & \mathbf{B}^{\mathbf{T}} & \mathbf{C}^{\mathbf{T}} \\
\mathbf{B} & \mathbf{0} & \mathbf{0} \\
\mathbf{C} & \mathbf{0} & \mathbf{0}
\end{array}\right)
$$


while for the Kelvin-Voigt model, we have:

$$
\mathbf{D}_{1}=\left(\begin{array}{cccc}
\mathbf{A}_{1} & 0 & 0 & 0 \\
0 & 0 & 0 & 0 \\
0 & 0 & 0 & 0 \\
0 & 0 & 0 & 0
\end{array}\right) \quad \mathbf{D}_{0}=\left(\begin{array}{cccc}
0 & 0 & \mathbf{B}^{\mathrm{T}} & \mathbf{C}^{\mathrm{T}} \\
\mathbf{0} & \mathbf{A}_{0} & \mathbf{B}^{\mathrm{T}} & \mathbf{C}^{\mathrm{T}} \\
\mathbf{B} & \mathbf{B} & \mathbf{0} & \mathbf{0} \\
\mathbf{C} & \mathbf{C} & \mathbf{0} & \mathbf{0}
\end{array}\right)
$$

The Maxwell system is a differential algebraic equation of index two, while the Kelvin-Voigt system in its current form is of index one.

The discrete inf-sup conditions (4.1), (4.2) guarantee that the matrices $\mathbf{A}_{\mathbf{0}}, \mathbf{A}_{\mathbf{1}}$ and $\mathbf{B}, \mathbf{C}$ are such that $\operatorname{det}\left(\lambda \mathbf{D}_{\mathbf{1}}+\mathbf{D}_{\mathbf{0}}\right) \not \equiv 0$ as a function of $\lambda$ for either system. Hence, with the spatial discretizations of Section 4, the systems will be regular, and therefore solvable. The already well-developed theory of discretizations of DAEs therefore carries over to these systems.

\section{NumERICAL EXPERIMENTS}

We now turn to consider a series of numerical experiments for both models in two dimensions. We shall focus on two aspects. First, we demonstrate convergence rates for different material parameter values on the unit square with constructed exact solutions. Second, we give examples of viscoelastic behaviour through looking at simple creep and relaxation scenarios. All simulations have been run using the DOLFIN library [27] from the FEniCS project [22].

The spatial discretization is based on the family of elasticity elements defined by (4.6), and its variant (4.7). The temporal discretization is carried out using the L-stable, second-order accurate, TR- $\mathrm{BDF}_{2}$ scheme, that is, a trapezoidal rule followed by a 2-step backward difference scheme at each time step [9]. For the general DAE (5.1), this scheme takes the following form for $n=0,1, \ldots$ :

$$
\begin{aligned}
& \mathbf{D}_{\mathbf{1}}\left(\mathbf{y}^{n+\frac{1}{2}}-\mathbf{y}^{n}\right)=\frac{1}{2} \frac{\Delta t}{2}\left(\mathbf{f}^{n+\frac{1}{2}}+\mathbf{f}^{n}-\mathbf{D}_{\mathbf{0}}\left(\mathbf{y}^{n+\frac{1}{2}}+\mathbf{y}^{n}\right)\right) \\
& \left(\frac{\Delta t}{2}\right)^{-1} \mathbf{D}_{\mathbf{1}}\left(\frac{3}{2} \mathbf{y}^{n+1}-2 \mathbf{y}^{n+\frac{1}{2}}+\frac{1}{2} \mathbf{y}^{n}\right)+\mathbf{D}_{\mathbf{0}} \mathbf{y}^{n+1}=\mathbf{f}^{n+1}
\end{aligned}
$$

The linear systems of equations that result from the application of this scheme to $\mathbf{D}_{\mathbf{0}}, \mathbf{D}_{\mathbf{1}}$ defined by (5.2) and (5.3), share a common matrix structure and again the discrete inf-sup conditions guarantee the solvability at each time step for any fixed $\Delta t>0$. On the other hand, it is not immediately evident that the time discretizations are stable uniformly in $h$. However, there are no indications in the following convergence experiments of that stability not holding uniformly.

6.1. Convergence. The families of finite element spaces defined by (4.6) and (4.7) were suggested and analyzed for the elasticity equations by Arnold et al [6] and Falk [20] respectively. As these have not been widely used for numerical simulations, we commence by examining their convergence properties for the elasticity equations separately. We continue by considering the finite element spaces (4.6) with $k=2$ in space and the scheme (6.1) in time for the Maxwell and Kelvin-Voigt models.

6.1.1. Elasticity. Consider a discretization of the isotropic elasticity equations (3.6) using the element spaces $\Sigma_{h, k}, V_{h, k}$ and $Q_{h, k}$ as defined by (4.6). We let $\Omega=[0,1]^{2}$ and consider a uniform, regular triangulation of $\Omega$. We solve for the following displacement field over $\Omega$ :

$$
u\left(x_{0}, x_{1}\right)=\left(\begin{array}{c}
-x_{1} \sin \left(\pi x_{0}\right) \\
\frac{1}{2} \pi x_{1}^{2} \cos \left(\pi x_{0}\right)
\end{array}\right)
$$

with corresponding elastic stress $\sigma=2 \mu \varepsilon(u)+\lambda \operatorname{div} u I$ and force $g=\operatorname{div} \sigma$. Observe that $u$, as defined by (6.2), is divergence-free and hence that the stress $\sigma$ and force 
$g$ are independent of $\lambda$. The convergence rates for the displacement and stress approximations using $\mu=1, \lambda=100, k=1,2,3$ are given in Table 1 and are in agreement with the predicted rates.

\begin{tabular}{|c|c|c|c|c|}
\hline$k$ & $\log _{2} \frac{\left\|u-u_{h}\right\|_{0}}{\left\|u-u_{h / 2}\right\|_{0}}$ & $\log _{2} \frac{\left\|\sigma-\sigma_{h}\right\|_{0}}{\left\|\sigma-\sigma_{h / 2}\right\|_{0}}$ & $\log _{2} \frac{\left\|\sigma-\sigma_{h}\right\|_{\text {div }}}{\left\|\sigma-\sigma_{h / 2}\right\|_{\text {div }}}$ & $\log _{2} \frac{\left\|\gamma-\gamma_{h}\right\|_{0}}{\left\|\gamma-\gamma_{h / 2}\right\|_{0}}$ \\
\hline 1 & 1 & 0.997 & 1 & 1.01 \\
2 & 2 & 1.99 & 2 & 2 \\
3 & 3 & 3 & 3 & 3 \\
\hline
\end{tabular}

TABLE 1. Convergence rates for elasticity using $\Sigma_{h, k} \times V_{h, k} \times Q_{h, k}$, $h=(16)^{-1}, k=1,2,3$, for $\lambda=100, \mu=1$. The exact displacement $u$ is defined by $(6.2)$.

Furthermore, if the displacement is small in comparison to the stress, improved convergence in the $L^{2}$-norm of stress has been observed. With the exact solution,

$$
u\left(x_{0}, x_{1}\right)=\left(\begin{array}{l}
\sin \left(\pi x_{0}\right) \sin \left(\pi x_{1}\right) \\
\sin \left(\pi x_{0}\right) \sin \left(\pi x_{1}\right)
\end{array}\right)
$$

and $\mu=1, \lambda=1000$, the convergence rates for $k=1$ are given in Table 2. Observe that the $L^{2}$ error rate of the stress is consistently close to 2 , that is, of one order higher than predicted by the analysis. This might be attributed to the inconsistently high convergence rates for the rotation and also to the large difference in magnitude of the stress and the rotation; The stress is many orders larger than the rotation in this test case and so the error contribution from the rotation will be less significant.

\begin{tabular}{|c|c|c|c|c|}
\hline$h^{-1}$ & $\log _{2} \frac{\left\|u-u_{h}\right\|_{0}}{\left\|u-u_{h / 2}\right\|_{0}}$ & $\log _{2} \frac{\left\|\sigma-\sigma_{h}\right\|_{0}}{\left\|\sigma-\sigma_{h / 2}\right\|_{0}}$ & $\log _{2} \frac{\left\|\sigma-\sigma_{h}\right\|_{\text {div }}}{\left\|\sigma-\sigma_{h / 2}\right\|_{\text {div }}}$ & $\log _{2} \frac{\left\|\gamma-\gamma_{h}\right\|_{0}}{\left\|\gamma-\gamma_{h / 2}\right\|_{0}}$ \\
\hline 4 & 2.82 & 1.91 & 0.98 & 2.80 \\
8 & 2.45 & 1.97 & 0.995 & 2.88 \\
16 & 1.42 & 1.99 & 0.999 & 2.68 \\
32 & 1.04 & 2.00 & 1.00 & 1.74 \\
\hline
\end{tabular}

TABLE 2. Convergence rates for elasticity using $\Sigma_{h, 1} \times V_{h, 1} \times Q_{h, 1}$, for $\lambda=1000, \mu=1$. The exact displacement $u$ is defined by (6.3).

In order to demonstrate the convergence properties of the element spaces of Falk (4.7), we replace $Q_{h, k}$ by $\tilde{Q}_{h, k}$. Solving for the divergence free displacement of (6.2) with $\mu=1, \lambda=100$, gives the convergence rates in Table 3 . The anticipated improvement of the $L^{2}$ convergence for the stress and rotation is observed.

\begin{tabular}{|c|c|c|c|c|}
\hline$k$ & $\log _{2} \frac{\left\|u-u_{h}\right\|_{0}}{\left\|u u-u_{h / 2}\right\|_{0}}$ & $\log _{2} \frac{\left\|\sigma-\sigma_{h}\right\|_{0}}{\left\|\sigma-\sigma_{h / 2}\right\|_{0}}$ & $\log _{2} \frac{\left\|\sigma-\sigma_{h}\right\|_{\text {div }}}{\| \sigma-\sigma_{h} / 2} \|_{\text {div }}$ & $\log _{2} \frac{\left\|\gamma-\gamma_{h}\right\|_{0}}{\left\|\gamma-\gamma_{h} / 2\right\|_{0}}$ \\
\hline 1 & 1 & 2 & 1 & 2 \\
2 & 2 & 3 & 2 & 3.17 \\
3 & 3 & 4.03 & 3.04 & 4.05 \\
\hline
\end{tabular}

TABLE 3. Convergence rates for elasticity using $\Sigma_{h, k} \times V_{h, k} \times \tilde{Q}_{h, k}$, $h=(16)^{-1}, k=1,2,3$, for $\lambda=100, \mu=1$. The exact displacement $u$ is defined by $(6.2)$. 
6.1.2. Maxwell. We continue letting $\Omega=[0,1]^{2}$, consider a regular triangulation $\mathcal{T}_{h}$ of $\Omega$ and the associated second order finite element spaces of the family (4.6): $\Sigma_{h, 2}, V_{h, 2}$ and $Q_{h, 2}$. Moreover, we let $T=1$ and consider a uniform partition of the time domain $[0, T]$ and the $\mathrm{TR}-\mathrm{BDF}_{2}$ difference scheme defined by (6.1). We shall let $\mu_{0}$ dominates $\mu_{1}$ and solve for a known smooth velocity in order to demonstrate robustness with regard to these parameters.

Define

$$
v(x, t)=e^{t-1}\left(\left(2 \mu_{1}\right)^{-1} 2 t+\left(2 \mu_{0}\right)^{-1} t^{2}\right)\left(\begin{array}{c}
-x_{1} \sin \left(\pi x_{0}\right) \\
\frac{1}{2} \pi x_{1}^{2} \cos \left(\pi x_{0}\right)
\end{array}\right)
$$

and let $\lambda_{0}=\lambda_{1}=100, \mu_{0}=100$ and $\mu_{1}=1$. Observe that the consistency condition at the initial time is satisfied, in particular that $\sigma(x, t)=0$ at $t=0$. The convergence rates at $T=1.0$ are given in Table 4 . We conclude that there is no loss of convergence even though $\mu_{0}>>\mu_{1}$.

\begin{tabular}{|c|c|c|c|c|}
\hline$h^{-1}$ & $\log _{2} \frac{\left\|v-v_{h}\right\|_{0}}{\left\|v-v_{h / 2}\right\|_{0}}$ & $\log _{2} \frac{\left\|\sigma-\sigma_{h}\right\|_{0}}{\left\|\sigma-\sigma_{h / 2}\right\|_{0}}$ & $\log _{2} \frac{\left\|\sigma-\sigma_{h}\right\|_{\text {div }}}{\left\|\sigma-\sigma_{h / 2}\right\|_{\text {div }}}$ & $\log _{2} \frac{\left\|\gamma-\gamma_{h}\right\|_{0}}{\left\|\gamma-\gamma_{h / 2}\right\|_{0}}$ \\
\hline 4 & 1.96 & 2.03 & 1.99 & 1.98 \\
8 & 1.98 & 2 & 2 & 1.99 \\
16 & 1.99 & 1.99 & 2 & 1.99 \\
\hline
\end{tabular}

TABLE 4. Maxwell convergence rates for $\lambda_{0}=\lambda_{1}=100, \mu_{0}=100$, $\mu_{1}=1$ at $T=1 . \Delta t=h$. Exact velocity $v$ as defined by $(6.4)$

6.1.3. Kelvin-Voigt. For the Kelvin-Voigt model, we consider the previous domain in time and space, but solve for $v$ given by (6.5).

$$
v(x, t)=\left(2 t+t^{2}\right) e^{t-1}\left(\begin{array}{l}
\sin \left(\pi x_{0}\right) \sin \left(\pi x_{1}\right) \\
\sin \left(\pi x_{0}\right) \sin \left(\pi x_{1}\right)
\end{array}\right)
$$

and enforce the initial condition $\sigma_{1}(x, 0)=0$. The $L^{2}$ error of the velocity and rotation and $H$ (div) error of the stresses, are measured at each time step and included in Table 5 . We observe that the convergence rates point-wise in time is of the order $\mathcal{O}\left(h^{2}\right)$.

\begin{tabular}{|c|c|c|c|c|}
\hline$h^{-1}$ & $\log _{2} \frac{\left\|v-v_{h}\right\|_{0}}{\left\|v-v_{h / 2}\right\|_{0}}$ & $\log _{2} \frac{\left\|\sigma_{0}-\sigma_{0, h}\right\|_{\text {div }}}{\left\|\sigma_{0}-\sigma_{0, h / 2}\right\|_{\text {div }}}$ & $\log _{2} \frac{\left\|\sigma_{1}-\sigma_{1, h}\right\|_{\text {div }}}{\left\|\sigma_{1}-\sigma_{1, h / 2}\right\|_{\text {div }}}$ & $\log _{2} \frac{\left\|\gamma-\gamma_{h}\right\|_{0}}{\left\|\gamma-\gamma_{h / 2}\right\|_{0}}$ \\
\hline 4 & 1.98 & 1.98 & 1.93 & 2.14 \\
8 & 1.99 & 1.99 & 1.97 & 2.06 \\
16 & 2 & 2 & 1.98 & 2.02 \\
\hline
\end{tabular}

TABLE 5. Kelvin-Voigt convergence rates for $\lambda_{0}=\lambda_{1}=100, \mu_{0}=$ 100, $\mu_{1}=1$ at $T=1.0 . \Delta t=h$. Exact solutions corresponding to exact velocity defined by $(6.5)$.

6.2. Relaxation and creep. We now turn to illustrate the two main viscoelastic behaviour characteristics, creep and relaxation. The Maxwell model exhibits stress relaxation as response to non-zero displacement and the Kelvin-Voigt model exhibits creep as response to applied traction. Thus, we shall qualitatively demonstrate a relaxation pattern using the Maxwell model and a creep pattern with the Kelvin-Voigt model. However, the simplest model that predicts both adequate stress relaxation and creep, is the standard linear solid (2.10). For this reason, and 


\begin{tabular}{|c|c|c|}
\hline Maxwell & Kelvin-Voigt & Standard linear solid \\
\hline$\mu_{0}=20.0, \lambda_{0}=100.0$ & $\mu_{0}=20.0, \lambda_{0}=100.0$ & $\mu_{0}^{0}=5.0, \lambda_{0}^{0}=100.0$ \\
$\mu_{1}=80.0, \lambda_{1}=100.0$ & $\mu_{1}=80.0, \lambda_{1}=100.0$ & $\mu_{1}^{0}=30.0, \lambda_{1}^{0}=100.0$ \\
& & $\mu_{1}^{1}=20.0, \lambda_{1}^{1}=100.0$ \\
\hline
\end{tabular}

TABLE 6. Parameters values used in the creep and relaxation simulations. The parameters $\mu_{i}^{(j)}$ and $\lambda_{i}^{(j)}$ correspond to the compliance tensors $A_{i}^{(j)}$ of the separate models.
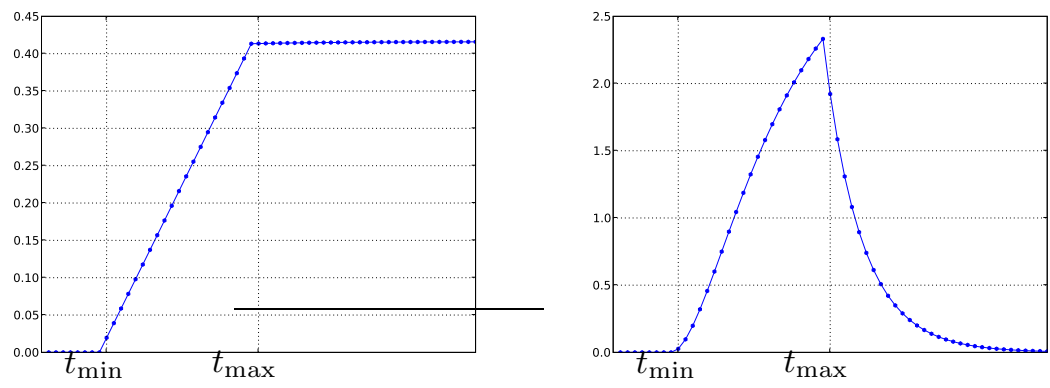

Figure 1. Maxwell response to applied velocity. Left: Displacement $u$ versus time $t$. Right: Stress $\|\sigma(t)\|_{A_{1}}$ versus time $t$. Discretization parameters: $\Delta t=1.0 / 30, h=1.0 / 32, t_{\min }=0.3$, $t_{\max }=1.0, T=2.0$.

in order to show the numerical method applied to a more general model, we also include experiments for these equations.

For the experiments, we consider the usual domain in space: $\left(x_{0}, x_{1}\right) \in \Omega=$ $[0,1]^{2}$ and $t \in[0, T]$. We let the body be clamped at the left boundary $x_{0}=0$ and stress-free at the top and bottom $x_{1}=0,1$. On the right boundary, a velocity is prescribed for the relaxation experiments and a prescribed traction is used for the creep experiments. The material parameters used are listed in Table 6 .

6.2.1. Relaxation. The Maxwell and standard linear solid models exhibit stress relaxation as response to non-zero displacement. To illustrate this behaviour, we apply a unit boundary velocity in the $x_{0}$ direction, on the right boundary $x_{0}=1$ at a given time $t_{\min }$ and remove the velocity at $t_{\max }$. This produces a displacement, in particular a constant displacement for $t>t_{\max }$. The Maxwell model predicts that the stresses increase from $t_{\min }$ to $t_{\max }$ and after that decrease to the zero limit. For the standard linear solid model, the stresses will decrease toward an elastic limit for $t>t_{\max }$.

The displacement and stress approximations for the Maxwell model are plotted in Figure 1. Observe in particular the exponential relaxation of the stress for $t>t_{\max }$. The same experiment for the standard linear solid is plotted in Figure 2.

6.2.2. Creep. The Kelvin-Voigt and standard linear solid models predict creep as response to applied traction. To demonstrate this, we apply a unit boundary traction in the $x_{0}$ direction at the right boundary $x_{0}=1$ at a given time $t_{\min }$ and remove at $t_{\max }$. The $L^{2}(\Omega)$ norms of the approximated displacements and stresses are displayed in Figure 3 for the Kelvin-Voigt model and Figure 4 for the standard linear solid. Observe the initial increase towards the elastic limit and subsequent 

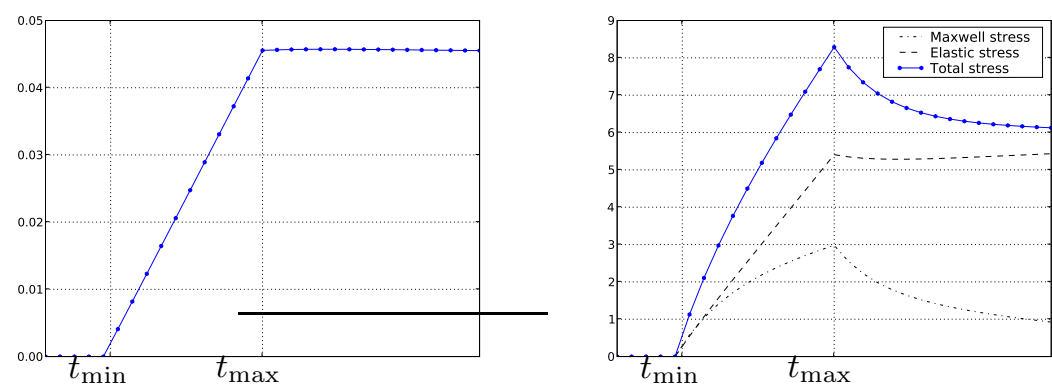

FiguRE 2. Standard linear solid response to applied velocity. Left: Displacement $u$ versus time $t$. Right: Stresses $\left\|\sigma_{0}(t)\right\|$, $\left\|\sigma_{1}(t)\right\|$, and $\|\sigma(t)\|_{0}$ versus time $t$. Discretization parameters: $\Delta t=1.0 / 30, h=1.0 / 32, t_{\min }=0.3, t_{\max }=1.0, T=2.0$. (The applied velocity is one tenth of the one applied for the Maxwell model.) The standard linear model can, in term of springs and dashpots, be viewed as a Maxwell element in parallel with an elastic spring. The corresponding components of the stress are therefore labelled Maxwell and Elastic according to this interpretation.
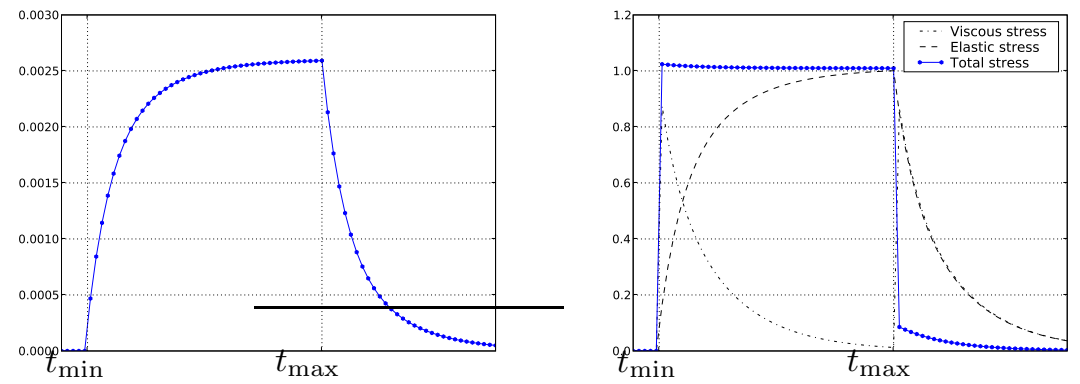

Figure 3. Kelvin-Voigt response to applied traction. Left: Displacement $u$ versus time $t$. Right: Viscous and elastic stress, $\left\|\sigma_{0}(t)\right\|_{0}$ and $\left\|\sigma_{1}(t)\right\|_{0}$ versus time $t$. Parameters: $\Delta t=1.0 / 30$, $h=1.0 / 32, t_{\min }=0.3, t_{\max }=3.0, T=5.0$.

decrease for the displacement for the Kelvin-Voigt model. Also note the instantaneous displacements for the standard linear solid.

\section{CONClusion}

We have presented a unified mixed finite element framework for linear viscoelasticity. The framework relies on mixed finite element discretizations originating from linear elasticity. These discretizations have been evaluated by numerical experiments both for linear elasticity and viscoelasticity. The analytical predictions are confirmed and the anticipated robustness with regard to material parameters demonstrated.

Acknowledgments. The authors acknowledge and are grateful for the initiative and valuable advice provided by Hans Petter Langtangen. 

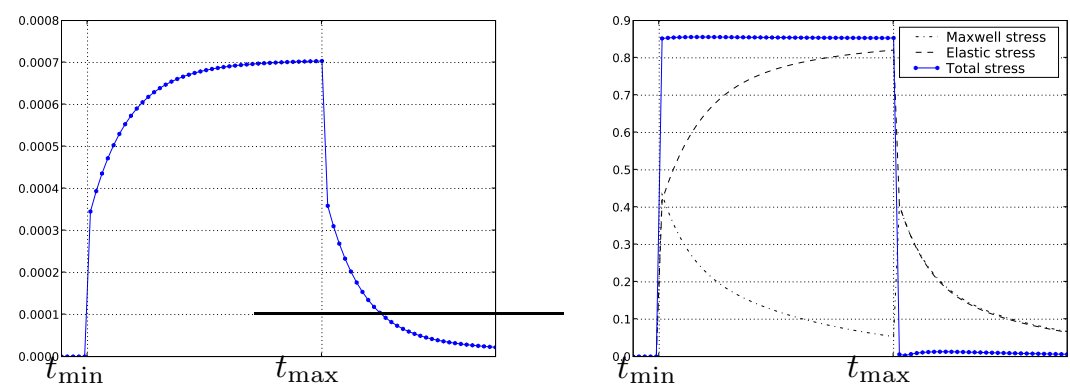

FiguRE 4. Standard linear solid response to applied traction. Left: Displacement $u$ versus time $t$. Right: Maxwell, elastic and total stress, $\left\|\sigma_{0}(t)\right\|_{0},\left\|\sigma_{1}(t)\right\|_{0}$ and $\|\sigma(t)\|_{0}$ versus time $t$. Parameters: $\Delta t=1.0 / 30, h=1.0 / 32, t_{\min }=0.3, t_{\max }=3.0, T=5.0$. (The applied traction is ten times the one applied for the Kelvin-Voigt model.)

\section{REFERENCES}

[1] S. Adams and B. Cockburn. A mixed finite element method for elasticity in three dimensions. J. Sci. Comput., 25:515-521, 2005.

[2] K. Adolfsson, M. Enelund, S. Larsson, and M. Racheva. Discretization of integro-differential equations modeling dynamic fractional order viscoelasticity. In Proceedings of Large-Scale Scientific Computations, 2005, Sozopol, Bulgaria, pages 76-83. Springer, 2006.

[3] M. Amara and J. M. Thomas. Equilibrium finite elements for the linear elastic problem. Numer. Math., 33:367-383, 1979.

[4] D. N. Arnold, G. Awanou, and R. Winther. Finite elements for symmetric tensors in three dimensions. Math. Computation, 77:1229-1251, 2008.

[5] D. N. Arnold, F. Brezzi, and J. Douglas. PEERS: A new mixed finite element for plane elasticity. Japan J. Appl. Math., 1:347-367, 1984.

[6] D. N. Arnold, R. Falk, and R. Winther. Mixed finite element methods for linear elasticity with weakly imposed symmetry. Math. Computation, 76:1699-1723, 2007.

[7] D. N. Arnold, R. S. Falk, and R. Winther. Finite element exterior calculus, homological techniques and applications. Acta numerica, 2006.

[8] D. N. Arnold and R. Winther. Mixed finite elements for elasticity. Numer. Math., 92:401-419, 2002.

[9] R. Bank, W. Coughran, W. Fichtner, E. Grosse, D. Rose, and R. Smith. Transient simulation of silicon devices and circuits. IEEE Transactions on Computer-Aided Design on Integrated Circuits and Systems, 4:436-451, 1985.

[10] E. Becache, A. Ezziani, and P. Joly. A mixed finite element appproach for viscoelastic wave propagation. Computational Geosciences, 8:255-299, 2004.

[11] D. Braess. Finite elements. Theory, fast solvers, and applications in solid mechanics. Cambridge University Press, second edition, 2001.

[12] K. E. Brenan, S. L. Campbell, and L. R. Petzold. Numerical Solution of Initial-Value Problems in Differential-Algebraic Equations. SIAM, second edition, 1996.

[13] F. Brezzi. On the existence, uniqueness and approximation of saddle-point problems arising from Lagrangian multipliers. R.A.I.R.O. Anal. Numer., 2:129-151, 1974.

[14] F. Brezzi, J. Douglas, and L. D. Marini. Two families of mixed elements for second order elliptic problems. Numer. Math., 47:217-235, 1985.

[15] S. H. Christiansen and R. Winther. Smoothed projections in finite element exterior calculus. Math. computation, 77:813-829, 2008.

[16] R. Dautray and J.-L. Lions. Mathematical Analysis and Numerical Methods for Science and Technology, volume 1. Springer-Verlag, 1990.

[17] A. D. Drozdov. Mechanics of Viscoelastic Solids. Wiley, 1998.

[18] L. C. Evans. Partial Differential Equations. American Mathematical Society, 2002.

[19] M. Fabrizio and A. Morro. Mathematical Problems in Linear Viscoelasticity. SIAM, 1992.

[20] R. S. Falk. Finite element methods for linear elasticity. In Mixed Finite Elements, Compatibility Conditions and Applications. Springer, 2008. 
[21] M. Farhloul and M. Fortin. Dual hybrid methods for the elasticity and the stokes problem: a unified approach. Numer. Math., 76:417-440, 1997.

[22] FEniCS. FEniCS project. URL: http//www.fenics.org/, 2007.

[23] J. D. Ferry. Viscoelastic Properties of Polymers. John Wiley and Sons, 2002.

[24] B. M. Fraijs de Veubeke. Stress function approach. In Proc. of the World Congress on Finite Element Methods in Structural Mechanics, volume 1, pages J.1-J.51, 1975.

[25] M. E. Gurtin and E. Sternberg. On the linear theory of viscoelasticity. Arch. Rational Mech. Anal, 11:291-356, 1962.

[26] E. Hairer and G. Wanner. Solving Ordinary Differential Equations II. Stiff and DifferentialAlgebraic Problems. Springer, second edition, 1996.

[27] J. Hoffman, J. Jansson, A. Logg, G. N. Wells, et al. DOLFIN. URL: http//www.fenics.org/wiki/DOLFIN, 2006

[28] A. Idesman, R. Niekamp, and E. Stein. Finite elements in space and time for generalized viscoelastic Maxwell model. Computational Mechanics, 27:49-60, 2001.

[29] A. R. Johnon and A. Tessler. A viscoelastic higher-order beam finite element. In J. R. Whiteman, editor, The Mathematics of Finite Elements and Applications, pages 333-345. Wiley, 1997.

[30] P. Le Tallec and M. Ravachol. Finite element approximation of viscoelastic progressively incompressible flows. Numerische Mathematik, 56:43-66, 1989.

[31] J. C. Nédélec. A new family of mixed finite elements in $\mathbf{R}^{3}$. Numer. Math., 50:57-81, 1986.

[32] N. Phan-Thien. Understanding Viscoelasticity. Basics of Rheology. Springer, 2002.

[33] B. Rivière, S. Shaw, and J. R. Whiteman. Discontinuous Galerkin finite element methods for linear elasticity and quasistatic linear viscoelasticity. Numer. Math, 95:347-376, 2003.

[34] B. Rivière, S. Shaw, and J. R. Whiteman. Discontinuous Galerkin finite element methods for dynamic linear solid viscoelasticity problems. Numerical Methods for Partial Differential Equations, 23:1149-1166, 2007.

[35] S. Shaw. Finite element and discrete time methods for continuum problems with memory and applications to viscoelasticity. PhD thesis, Department of Mathematics and Statistics, Brunel University, 1993.

[36] S. Shaw, M. K. Warby, J. R. Whiteman, C. Dawson, and M. F. Wheeler. Numerical techniques for the treatment of quasistatic solid viscoelastic stress problems. Comput. Methods Appl. Mech. Engrg, 118:211-237, 1994.

[37] S. Shaw and J. R. Whiteman. Numerical solution of linear quasistatic hereditary viscoelasticity problems. Siam J. Numer. Anal., 38:80-97, 2000.

[38] S. Shaw and J. R. Whiteman. A posteriori error estimates for space-time finite element approximation of quasistatic hereditary linear viscoelasticity problems. Comput. Methods Appl. Mech. engrg., 193:5551-5572, 2004.

[39] R. Stenberg. A family of mixed finite elements for the elasticity problem. Numer. Math., 53:513-538, 1988 .

[40] N. Tschoegl. The Phenomenological theory of linear viscoelastic behaviour. Springer-Verlag, first edition, 1989.

Centre of Mathematics for Applications, P.O.Box 1053, 0316 Oslo, Norway

E-mail address: meg@cma.uio.no

Centre of Mathematics for Applications, P.O.Box 1053, 0316 Oslo, Norway

E-mail address: ragnar.winther@cma.uio.no 